\title{
FINITE VOLUME SCHEMES ON LORENTZIAN MANIFOLDS*
}

\author{
PAULO AMORIM ${ }^{\dagger}$, PHILIPPE G. LEFLOCH ${ }^{\ddagger}$ AND BAVER OKUTMUSTUR ${ }^{\S}$
}

\begin{abstract}
We investigate the numerical approximation of (discontinuous) entropy solutions to nonlinear hyperbolic conservation laws posed on a Lorentzian manifold. Our main result establishes the convergence of monotone and first-order finite volume schemes for a large class of (space and time) triangulations. The proof relies on a discrete version of entropy inequalities and an entropy dissipation bound, which take into account the manifold geometry and were originally discovered by Cockburn, Coquel, and LeFloch in the (flat) Euclidian setting.
\end{abstract}

Key words. Conservation law, Lorenzian manifold, entropy condition, measure-valued solution, finite volume scheme, convergence analysis.

AMS subject classifications. Primary: 35L65; Secondary: 76L05, 76N.

\section{Introduction}

We are interested in discontinuous solutions to nonlinear hyperbolic conservation laws posed on a globally hyperbolic Lorentzian manifold, and we introduce a class of first-order, monotone finite volume schemes which enjoy geometrically natural stability properties. In turn, we conclude that the proposed finite volume schemes converge (in a strong topology) toward entropy solutions to hyperbolic conservation laws. Recall that the well-posedness theory for nonlinear hyperbolic equations posed on a manifold was recently established by Ben-Artzi and LeFloch [2] and LeFloch and Okutmustur $[17,18]$. On the other hand, our proof of convergence of the finite volume method can be viewed as a generalization to Lorentzian manifolds of the technique introduced by Cockburn, Coquel and LeFloch $[5,7]$ for the (flat) Euclidean setting and already extended to Riemannian manifolds by Amorim, Ben-Artzi, and LeFloch [1].

Major conceptual and technical difficulties arise in the analysis of partial differential equations posed on a Lorentzian manifold. Several new difficulties also appear when trying to generalize the convergence results in $[1,5,7]$ to Lorentzian manifolds. Most importantly, a space and time triangulation must be introduced and the geometry of the manifold must be taken into account in the discretization. We point out that, on a Lorentzian manifold, one cannot canonically choose a preferred foliation by spacelike hypersurfaces in general, so that it is important for the discretization to be robust enough to allow for a large class of foliations and of spacetime triangulations. From the numerical analysis standpoint, it is challenging to design and analyze discretization schemes that are consistent with the geometry of the given manifold. Our guide in deriving the necessary estimates was to ensure that all of our arguments are intrinsic in nature, and thus do not explicitly rely on a choice of local coordinates.

The main assumption of global hyperbolicity made on the given Lorentzian background is natural, and ensures that the manifold enjoys reasonable causality properties. Furthermore, the class of schemes considered in the present paper is quite general

\footnotetext{
*Received: July 20, 2008; accepted: Octber 20, 2008. Communicated by Shi Jin.

${ }^{\dagger}$ Laboratoire Jacques-Louis Lions \& Centre National de la Recherche Scientifique, Université Pierre et Marie Curie (Paris 6), 4 Place Jussieu, 75252 Paris, France (Pauloamo@gmail.com).

${ }^{\ddagger}$ Laboratoire Jacques-Louis Lions \& Centre National de la Recherche Scientifique, Université Pierre et Marie Curie (Paris 6), 4 Place Jussieu, 75252 Paris, France (pgLeFloch@gmail.com).

$\S$ Laboratoire Jacques-Louis Lions \& Centre National de la Recherche Scientifique, Université Pierre et Marie Curie (Paris 6), 4 Place Jussieu, 75252 Paris, France (Okutmustur@ann.jussieu.fr).
} 
and, essentially, requires that the numerical flux functions are monotone. It encompasses a large class of spacetime triangulations, in which the elements may become degenerate (in the limit) in the spatial direction.

More specifically, we show here that the proposed finite volume schemes can be expressed as a convex decomposition of essentially one-dimensional schemes, and we derive a discrete version of entropy inequalities as well as sharp estimates on the entropy dissipation. Strong convergence towards an entropy solution follows from DiPerna's uniqueness theorem [11].

For another approach to conservation laws on manifolds we refer to Panov [19] and for high-order numerical methods to Rossmanith, Bale, and LeVeque [20] and the references therein. DiPerna's measure-valued solutions were used to establish the convergence of schemes by Szepessy [21, 22], Coquel and LeFloch [8, 9, 10], and Cockburn, Coquel, and LeFloch [5, 7]. For many related results and a review about the convergence techniques for hyperbolic problems, we refer to Tadmor [23] and Tadmor, Rascle, and Bagneiri [24]. Further hyperbolic models, including also a coupling with elliptic equations, as well as many applications were successfully investigated by Kröner [13], and Eymard, Gallouet, and Herbin [12]. For higher-order schemes, see the paper by Kröner, Noelle, and Rokyta [14]. Also, an alternative approach to the convergence of finite volume schemes was proposed by Westdickenberg and Noelle [25]. Finally, note that Kuznetsov's error estimate, established in $[4,6]$ in the Euclidian setting, was recently extended to hyperbolic conservation laws on manifolds [16].

An outline of this paper follows. In section 2, we state some preliminary results from the theory of conservation laws on manifolds. In section 3, we introduce a class of finite volume schemes, and state the assumptions made on the discretization. Next, we state our main convergence result in Theorem 3.3. In section 4, we gather several important remarks and examples of particular interest. In section 5 , we derive various stability estimates, which are of independent interest and are also later used, in section 6 , to conclude with the convergence proof for the proposed schemes.

\section{Preliminaries on conservation laws on a Lorentzian manifold}

We will need some existence results, for which we refer to $[2,17,18]$. Let $(M, g)$ be a time-oriented, $(d+1)$-dimensional Lorentzian manifold. Here, $g$ is a metric with signature $(-,+, \ldots,+)$, and we recall that tangent vectors $X \in T_{p} M$ at a point $p \in M$ can be separated into timelike vectors $(g(X, X)<0)$, null vectors $(g(X, X)=0)$, and spacelike vectors $(g(X, X)>0)$. The manifold is assumed to be time-oriented, so that we can distinguish between past-oriented and future-oriented vectors. The Levi-Cevita connection associated to $g$ is denoted by $\nabla$ and, for instance, allows us to define the divergence operator $\operatorname{div}_{g}$. Finally we denote by $d V_{g}$ the volume element associated with the metric $g$.

Following [2], a flux-vector on a manifold is defined as a vector field $f=f(\bar{u}, p)$ depending on a real parameter $\bar{u}$, and the conservation law on $(M, g)$ associated with $f$ reads

$$
\operatorname{div}_{g}(f(u, p))=0, \quad u: M \rightarrow \mathbb{R} .
$$

Moreover, the flux-vector $f$ is said to be geometry compatible if

$$
\operatorname{div}_{g} f(\bar{u}, p)=0, \quad \bar{u} \in \mathbb{R}, p \in M,
$$

and to be timelike if its $u$-derivative is a timelike vector field

$$
g\left(\partial_{u} f(\bar{u}, p), \partial_{u} f(\bar{u}, p)\right)<0, \quad p \in M, \bar{u} \in \mathbb{R} .
$$


We are interested in the initial-value problem associated with (2.1). So, we fix a spacelike hypersurface $\mathcal{H}_{0} \subset M$ and a measurable and bounded function $u_{0}$ defined on $\mathcal{H}_{0}$. Then, we search for a function $u=u(p) \in L^{\infty}(M)$ satisfying $(2.1)$ in the distributional sense and such that the (weak) trace of $u$ on $\mathcal{H}_{0}$ coincides with $u_{0}$, that is,

$$
u_{\mid \mathcal{H}_{0}}=u_{0}
$$

It is natural to require that the vectors $\partial_{u} f(\bar{u}, p)$, which determine the propagation of waves in solutions of (2.1), are timelike and future-oriented.

We assume that the manifold $M$ is globally hyperbolic, in the sense that there exists a foliation of $M$ by spacelike, compact, oriented hypersurfaces $\mathcal{H}_{t}(t \in \mathbb{R})$ :

$$
M=\bigcup_{t \in \mathbb{R}} \mathcal{H}_{t}
$$

Any hypersurface $\mathcal{H}_{t_{0}}$ is referred to as a Cauchy surface in $M$, while the family of slices $\mathcal{H}_{t}(t \in \mathbb{R})$ is called an admissible foliation associated with $\mathcal{H}_{t_{0}}$. The future of the given hypersurface will be denoted by

$$
M_{+}:=\bigcup_{t \geq 0} \mathcal{H}_{t}
$$

Finally we denote by $n^{t}$ the future-oriented, normal vector field to each $\mathcal{H}_{t}$, and by $g^{t}$ the induced metric. Finally, along $\mathcal{H}_{t}$, we denote the normal component of a vector field $X$ by $X^{t}$, thus $X^{t}:=g\left(X, n^{t}\right)$. In the following, when there is no risk of confusion, we write $F(u)$ instead of $F(u, p)$.

Definition 2.1. A flux $F=F(\bar{u}, p)$ is called a convex entropy flux associated with the conservation law (2.1) if there exists a convex function $U: \mathbb{R} \rightarrow \mathbb{R}$ such that

$$
F(\bar{u}, p)=\int_{0}^{\bar{u}} \partial_{u} U\left(u^{\prime}\right) \partial_{u} f\left(u^{\prime}, p\right) d u^{\prime}, \quad p \in M, \bar{u} \in \mathbb{R} .
$$

A measurable and bounded function $u=u(p)$ is called an entropy solution of conservation law (2.1)-(2.2) if the following entropy inequality

$$
\begin{aligned}
& \int_{M_{+}} g\left(F(u), \nabla_{g} \phi\right) d V_{g}+\int_{M_{+}}\left(\operatorname{div}_{g} F\right)(u) \phi d V_{g} \\
& +\int_{\mathcal{H}_{0}} g_{0}\left(F\left(u_{0}\right), n_{0}\right) \phi_{\mathcal{H}_{0}} d V_{g_{0}}-\int_{M_{+}} U^{\prime}(u)\left(\operatorname{div}_{g} f\right)(u) \phi d V_{g} \geq 0
\end{aligned}
$$

holds for all convex entropy flux $F=F(\bar{u}, p)$ and all smooth functions $\phi \geq 0$ compactly supported in $M_{+}$.

In particular, the requirements in the above definition imply the inequality

$$
\operatorname{div}_{g}(F(u))-\left(\operatorname{div}_{g} F\right)(u)+U^{\prime}(u)\left(\operatorname{div}_{g} f\right)(u) \leq 0
$$

in the distributional sense. Next, denoting the space of integrable functions defined on the Riemannian slice $\left(\mathcal{H}_{t}, g_{t}\right)$ by $L_{g_{t}}^{1}\left(\mathcal{H}_{t}\right)$, from $[2,17,18]$ we recall the following result. 
THEOREM 2.2 (Well-posedness theory for conservation laws on a manifold). Consider a conservation law (2.1) posed on a globally hyperbolic Lorentzian manifold $M$ with compact slices. Let $\mathcal{H}_{0}$ be a Cauchy surface in $M$, and $u_{0}: \mathcal{H}_{0} \rightarrow \mathbb{R}$ be a function in $L^{\infty}\left(\mathcal{H}_{0}\right)$. Then the initial-value problem (2.1)-(2.4) admits a unique entropy solution $u=u(p) \in L_{\mathrm{loc}}^{\infty}\left(M_{+}\right)$. Moreover, for every admissible foliation $\mathcal{H}_{t}$ the trace $u_{\mid \mathcal{H}_{t}} \in L_{g_{t}}^{1}\left(\mathcal{H}_{t}\right)$ exists as a Lipschitz continuous function of $t$. When the flux is geometry compatible, the functions

$$
\left\|F^{t}\left(u_{\mid \mathcal{H}_{t}}\right)\right\|_{L_{g_{t}}^{1}\left(\mathcal{H}_{t}\right)}
$$

are non-increasing in time, for any convex entropy flux F. Moreover, given any two entropy solutions $u, v$, the function

$$
\left\|f^{t}\left(u_{\mid \mathcal{H}_{t}}\right)-f^{t}\left(v_{\mid \mathcal{H}_{t}}\right)\right\|_{L_{g_{t}}^{1}\left(\mathcal{H}_{t}\right)}
$$

is non-increasing in time.

Throughout the rest of this paper, a globally hyperbolic Lorentzian manifold is given, and we tackle the problem of the discretization of the initial value problem associated with the conservation law (2.1) and a given initial condition where $u_{0} \in$ $L^{\infty}\left(\mathcal{H}_{0}\right)$. In this paper, we do not assume that the flux $f$ is geometry compatible, and we refer to [18] for the generalization of the above theory. Throughout the present paper, we require the following growth condition: there exist constants $C_{1}, C_{2}>0$ such that for all $(\bar{u}, p) \in \mathbb{R} \times M$

$$
\left|\left(\operatorname{div}_{g} f\right)(\bar{u}, p)\right| \leq C_{1}+C_{2}|\bar{u}| .
$$

Two important remarks are in order.

- First of all, the terminology here differs from the one in the Riemannian (and Euclidean) cases, where the conservative variable is singled out. The class of conservation laws on a Riemannian manifold is recovered by taking $M=\mathbb{R} \times \widetilde{M}$, where $(\widetilde{M}, \bar{g})$ is a Riemannian manifold and $f(\bar{u}, p)=(\bar{u}, \bar{f}(\bar{u}, p)) \in$ $\mathbb{R} \times T_{p} \widetilde{M}$. We can then write $\operatorname{div}_{g}(f(u, p))=\partial_{t} u+\operatorname{div}_{\bar{g}}(\bar{f}(u, p))$.

- Second, in the Lorentzian case no time-translation property is available in general, contrary to the Riemannian case. Hence, no time-regularity is implied by the $L_{g_{t}}^{1}$ contraction property.

\section{Formulation and main result}

3.1. Definition of the finite volume schemes. Before we can state our main result, we must introduce some notation and motivate the formulation of the finite volume schemes under consideration. We consider a spacetime triangulation $\mathcal{T}^{h}=\bigcup_{K \in \mathcal{T}^{h}} K$ of the manifold $M_{+}$, which is made of (compact) spacetime elements $K$ and satisfies the following conditions:

- The boundary $\partial K$ of an element $K$ is a piecewise smooth $d$-dimensional manifold without a boundary, $\partial K=\bigcup_{e \subset \partial K} e$, and each $d$-dimensional element $e$ is a smooth manifold with piecewise smooth boundary and is either everywhere timelike or everywhere spacelike. The outward unit normal to $e \in \partial K$ is denoted by $\mathbf{n}_{K, e}$.

- Each element $K$ contains exactly two spacelike elements, with disjoint interiors, denoted by $e_{K}^{+}$and $e_{K}^{-}$, such that the outward unit normals to $K, \mathbf{n}_{K, e_{K}^{+}}$ and $\mathbf{n}_{K, e_{K}^{-}}$, are future- and past-oriented, respectively. They will be called the outflow and the inflow elements, respectively. 
- For each element $K$, the set of the lateral elements $\partial^{0} K:=\partial K \backslash\left\{e_{K}^{+}, e_{K}^{-}\right\}$is nonempty and timelike.

- For every pair of distinct elements $K, K^{\prime} \in \mathcal{T}^{h}$, the set $K \cap K^{\prime}$ is either a common element of $K, K^{\prime}$ or else a submanifold with dimension at most ( $d-$ 1).

- $\mathcal{H}_{0} \subset \bigcup_{K \in \mathcal{T}^{h}} \partial K$, where $\mathcal{H}_{0}$ is the initial Cauchy hypersurface.

- For every $K \in \mathcal{T}^{h}$, $\operatorname{diam} e_{K}^{ \pm} \leq h$.

For computing the diameter above, we assume that some reference Riemannian metric is fixed on the Lorentzian manifold; such a metric can be easily introduced from the expression of the Lorentzian metric (by replacing the signature $(-,+, \ldots,+$ ) by $(+,+, \ldots,+)$ in the expression in local coordinates). Given an element $K$, we denote the unique element distinct from $K$ sharing the element $e_{K}^{+}$(resp. $e_{K}^{-}$) with $K$ by $K^{+}$ (resp. $K^{-}$), and for each $e^{0} \in \partial^{0} K$, we denote the unique element sharing the element $e^{0}$ with $K$ by $K_{e^{0}}$. In addition, it is convenient to assume that the boundary of the element does not wildly "oscillate", in the sense that for all smooth vector fields $X$ defined on $e_{K}^{+}$,

$$
\left\|g_{p}\left(X(p), \mathbf{n}_{K, e_{K}^{+}}(p)\right)\right\|_{C^{2}\left(e_{K}^{+}\right)} \lesssim\|X(p)\|_{C^{2}\left(e_{K}^{+}\right)},
$$

where the implied constant (in $\lesssim$ ) is fixed throughout this paper. This condition is intended to rule out oscillations on the normal vector field due to the geometry of $e_{K}^{+}$. It restricts the variation of the normal on each element $e_{K}^{+}$, but not the variation from one element to the next.

The most natural way of introducing the finite volume method is to view the discrete solution as defined on the spacelike elements $e_{K}^{ \pm}$separating two elements. So, to a particular element $K$ we may associate two values, $u_{K}^{+}$and $u_{K}^{-}$associated to the unique outflow and inflow elements $e_{K}^{+}, e_{K}^{-}$. Then, one may determine that the value $u_{K}$ of the discrete solution on the element $K$ is the solution $u_{K}^{+}$determined on the inflow element $e_{K}^{-}$(one could just as well say that $u_{K}$ is the solution $u_{K}^{+}$determined on the outflow element $e_{K}^{+}$, or some average of the two, as long as one does this coherently throughout the manifold).

Thus, for any element $K$, integrate equation (2.1), apply the divergence theorem and decompose the boundary $\partial K$ into its parts $e_{K}^{+}, e_{K}^{-}$, and $\partial^{0} K$ :

$$
\begin{aligned}
& -\int_{e_{K}^{+}} g_{p}\left(f(u, p), \mathbf{n}_{K, e_{K}^{+}}(p)\right) d V_{g}-\int_{e_{K}^{-}} g_{p}\left(f(u, p), \mathbf{n}_{K, e_{K}^{-}}(p)\right) d V_{g} \\
& +\sum_{e^{0} \in \partial^{0} K} \int_{e^{0}} g_{p}\left(f(u, p), \mathbf{n}_{K, e^{0}}(p)\right) d p=0 .
\end{aligned}
$$

For any hypersurface $e \subset M$, we will often denote simply by $d V_{e}=d V_{g_{e}}$ the volume element of the induced metric $g_{e}$ associated with the Lorentzian metric $g$. Note the minus sign in the first two terms which comes from the fact that, for a Lorentzian manifold, the divergence theorem reads

$$
\int_{\Omega} \operatorname{div}_{g} f d V_{\Omega}=\int_{\partial \Omega} g(f, \tilde{\mathbf{n}}) d V_{\partial \Omega},
$$

in which $\tilde{\mathbf{n}}$ is the outward normal if it is spacelike, and the inward normal if it is timelike. This formula is nothing but the standard divergence theorem, with the signs of the normals properly taken into account. 
Given an element $K$, we want to compute an approximation $u_{K}^{+}$of the average of $u(p)$ in the outflow element $e_{K}^{+}$, given the values of $u_{K}^{-}$on $e_{K}^{-}$and of $u_{K_{e} 0}^{-}$for each $e^{0} \in \partial^{0} K$.

The following notation will be useful. Let $f$ be a flux on the manifold $M, K$ an element of the triangulation, and $e \subset \partial K$, respectively. Define the function $\mu_{K, e}^{f}: \mathbb{R} \rightarrow$ $\mathbb{R}$ by

$$
\mu_{K, e}^{f}(u):=\frac{1}{|e|} \int_{e} g_{p}\left(f(u, p), \mathbf{n}_{K, e}(p)\right) d V_{e}=\oint_{e} g_{p}\left(f(u, p), \mathbf{n}_{K, e}(p)\right) d V_{e},
$$

where $|e|$ is the measure of $e$. Also, if $w: M \rightarrow \mathbb{R}$ is a real-valued function, we write

$$
\mu_{e}^{w}:=\oint_{e} w(p) d V_{e}
$$

Using this notation, the second term in (3.2) is approximated by

$$
\int_{e_{K}^{-}} g_{p}\left(f(u, p), \mathbf{n}_{K, e_{K}^{-}}(p)\right) d V_{g} \simeq\left|e_{K}^{-}\right| \mu_{K, e_{K}^{-}}^{f}\left(u_{K}^{-}\right),
$$

and the last term is approximated by using

$$
\int_{e^{0}} g_{p}\left(f(u, p), \mathbf{n}_{K, e^{0}}(p)\right) d V_{e^{0}} \simeq\left|e^{0}\right| \mathbf{q}_{K, e^{0}}\left(u_{K}^{-}, u_{K_{e^{0}}}^{-}\right),
$$

where to each element $K$, and each element $e^{0} \in \partial^{0} K$ we associate a locally Lipschitz numerical flux function $\mathbf{q}_{K, e^{0}}(u, v): \mathbb{R}^{2} \rightarrow \mathbb{R}$ satisfying certain assumptions listed below.

Therefore, in view of the above approximation formulas we may write, as a discrete approximation of $(3.2)$,

$$
\left|e_{K}^{+}\right| \mu_{K^{+}, e_{K}^{+}}^{f}\left(u_{K}^{+}\right):=\left|e_{K}^{-}\right| \mu_{K, e_{K}^{-}}^{f}\left(u_{K}^{-}\right)-\sum_{e^{0} \in \partial^{0} K}\left|e^{0}\right| \mathbf{q}_{K, e^{0}}\left(u_{K}^{-}, u_{K_{e} 0}^{-}\right),
$$

which is the finite volume method of interest and, equivalently

$$
u_{K}^{+}:=\left(\mu_{K^{+}, e_{K}^{+}}^{f}\right)^{-1}\left(\frac{\left|e_{K}^{-}\right|}{\left|e_{K}^{+}\right|} \mu_{K, e_{K}^{-}}^{f}\left(u_{K}^{-}\right)-\sum_{e^{0} \in \partial^{0} K} \frac{\left|e^{0}\right|}{\left|e_{K}^{+}\right|} \mathbf{q}_{K, e^{0}}\left(u_{K}^{-}, u_{K_{e^{0}}}\right)\right) .
$$

The second formula which may be computed out numerically (using for instance a Newton algorithm) is justified by the following observation:

LEMMA 3.1. For any $K \in \mathcal{T}^{h}$, the function $u \mapsto \mu_{K, e_{K}^{-}}^{f}(u)$ is monotone increasing.

Proof. From (3.3) we deduce that

$$
\partial_{u} \mu_{K, e_{K}^{-}}^{f}(u)=\oint_{e} g_{p}\left(\partial_{u} f(u, p), \mathbf{n}_{K, e_{K}^{-}}(p)\right) d V_{e}>0
$$

since $\partial_{u} f(u, p)$ is future-oriented and $\mathbf{n}_{K, e_{K}^{-}}$is past-oriented.

Now, if $e_{K}^{-} \subset \mathcal{H}_{0}$, the initial condition (2.4) gives

$$
u_{K}^{-}:=\mu_{e_{K}^{-}}^{u_{0}}=\oint_{e_{K}^{-}} u_{0}(p) d V_{e_{K}^{-}} .
$$


Finally, we define the function $u^{h}: M \rightarrow \mathbb{R}$ by

$$
u^{h}(p):=u_{K}^{-}, \quad p \in K .
$$

On the other hand, for all $e \in \partial K$ we introduce the notation

$$
f_{e}(u, p):=g_{p}\left(f(u, p), \mathbf{n}_{K, e}(p)\right) .
$$

3.2. Assumptions on the numerical flux.

For the numerical flux $\mathbf{q}_{K, e^{0}}(u, v): \mathbb{R}^{2} \rightarrow \mathbb{R}$ we impose the following properties.

- Consistency property :

$$
\mathbf{q}_{K, e^{0}}(u, u)=\oint_{e^{0}} f_{e^{0}}(u, p) d V_{g}=\mu_{K, e^{0}}^{f}(u) .
$$

- Conservation property :

$$
\mathbf{q}_{K, e^{0}}(u, v)=-\mathbf{q}_{K_{e^{0}}, e^{0}}(v, u), \quad u, v \in \mathbb{R} .
$$

- Monotonicity property :

$$
\partial_{u} \mathbf{q}_{K, e^{0}}(u, v) \geq 0, \quad \partial_{v} \mathbf{q}_{K, e^{0}}(u, v) \leq 0 .
$$

For each element $K$, define the time-increment

$$
\tau_{K}=\frac{|K|}{\left|e_{K}^{+}\right|},
$$

where $|K|$ is the $((d+1)$-dimensional $)$ measure of $K$. We suppose that, as $h \rightarrow 0$,

$$
\tau:=\max _{K} \tau_{K} \rightarrow 0
$$

and

$$
\frac{h^{2}}{\min _{K} \tau_{K}} \rightarrow 0 .
$$

For stability purposes, we also impose the following $C F L$ condition, for all $K \in \mathcal{T}^{h}, e^{0} \in$ $\partial_{0} K$,

$$
\frac{\left|\partial^{0} K\right|}{\left|e_{K}^{+}\right|} \sup _{u \in \mathbb{R}}\left|\partial_{u} \mu_{K, e^{0}}^{f}(u)\right| \sup _{u \in \mathbb{R}} \partial_{u}\left(\mu_{K^{+}, e_{K}^{+}}^{f}\right)^{-1}(u) \leq 1 .
$$

3.3. Assumptions on the triangulation and main convergence result. For the triangulation $\mathcal{T}^{h}$, we will introduce an admissibility condition that is global and geometric in nature, and which is essentially optimal to ensure the convergence of the proposed schemes (see the following subsection for details). The condition only involves the time-evolution of the triangulation and is independent of the structure of the triangulation on spacelike elements. We stress that our method poses almost no restriction on the spacelike structure of the discretization.

The following notation will be used throughout this paper. Let $K \in \mathcal{T}^{h}$. We denote by $p_{K}^{0}$ and $p_{K}^{+}$the centers of mass of $\partial^{0} K$ and $e_{K}^{+}$, respectively. Note that $p_{K}^{0}$ does not lie on $\partial^{0} K$ (or even "close" to it), and that $p_{K}^{+}$does not necessarily lie 
on $e_{K}^{+}$. Next, we define the vector $\mathbf{w}_{K} \in T_{p_{K}^{+}} M$ as the vector at $p_{K}^{+}$tangent to the geodesic line connecting $p_{K}^{+}$to $p_{K}^{0}$ and with length $\operatorname{dist}\left(p_{K}^{+}, p_{K}^{0}\right)$ given by the reference Riemannian metric. This vector is well-defined if the discretization parameter $h$ is small enough.

We will also make the following assumption on the triangulation, namely that for $h$ sufficiently small, and for each element $e_{K}^{+}$, we may extend the normal vector field $\mathbf{n}_{K, e_{K}^{+}}$by parallel transporting it (using the metric structure on the manifold) to a neighborhood of $e_{K}^{+}$containing $p_{K}^{+}$. This is a natural assumption, which ensures that each element $e_{K}^{+}$of the triangulation tends to become flat in the limit.

Consider the quantity

$$
\mathcal{E}(K):=\frac{1}{\tau_{K}} \mathbf{w}_{K} \otimes \mathbf{n}_{K, e_{K}^{+}}
$$

which can be viewed as a quadratic form on $T_{p_{K}^{+}} M$. If $X, Y$ are two vectors at the point $p_{K}^{+}$, we have

$$
\mathcal{E}(K)(X, Y)=\frac{1}{\tau_{K}} \mathbf{w}_{K} \otimes \mathbf{n}_{K, e_{K}^{+}}(X, Y)=\frac{1}{\tau_{K}} g_{p_{K}^{+}}\left(X, \mathbf{w}_{K}\right) g_{p_{K}^{+}}\left(Y, \mathbf{n}_{K, e_{K}^{+}}\right) .
$$

We define the local deviation associated with $K, K^{-}$by

$$
|K| \mathcal{E}(K)-\left|K^{-}\right| \mathcal{E}\left(K^{-}\right),
$$

which measures the rate of change of the quantity $|K| \mathcal{E}(K)(X, Y)$ with respect to the timelike direction defined locally by the normals to the elements $e_{K}^{ \pm}$. Our admissibility criterion below requires that this rate of change should tend to zero with $h$ (after summation over all $K \in \mathcal{T}^{h}$ ).

Definition 3.2. A triangulation $\mathcal{T}^{h}$ is called an admissible triangulation if for every vector field $\Phi$ with compact support and every family of smooth vector fields $\Psi_{K}$, $K \in \mathcal{T}^{h}$ (each of them being defined on the manifold and associated with a given $K$ ), the local deviation satisfies

$$
\left|\sum_{K \in \mathcal{T}^{h}}\right| K\left|\mathcal{E}(K)\left(\Phi, \Psi_{K}\right)-\right| K^{-}\left|\mathcal{E}\left(K^{-}\right)\left(\Phi, \Psi_{K}\right)\right| \lesssim \eta(h)\|\Phi\|_{L^{\infty}} \sup _{K}\left\|\Psi_{K}\right\|_{L^{\infty}}
$$

for some fixed function $\eta(h)$ with $\eta(h) \rightarrow 0$.

Observe that, in (3.15), the vector $\mathbf{n}_{K, e_{K}^{+}}\left(p_{K}^{+}\right) \in T_{p_{K}^{+}} M$ makes sense, since the normal vector field was extended to include a neighborhood of $e_{K}^{+}$. The assumption (3.15) is a global geometric condition on the local deviation of the triangulation. As further discussed in section 4 below, this condition allows us to encompass a large class of implementable spacetime triangulations.

Finally, we are in a position to state the following theorem.

THEOREM 3.3 (Convergence of the finite volume schemes). Let $u^{h}$ be the sequence of functions generated by the finite volume method (3.4)-(3.7) on an admissible triangulation, with initial data $u_{0} \in L^{\infty}\left(\mathcal{H}_{0}\right)$, with numerical flux satisfying the conditions (3.9)-(3.11), and the CFL condition (3.14). Then, for every $T>0$ the sequence $u^{h}$ is uniformly bounded in $L^{\infty}\left(\bigcup_{t \in[0, T]} \mathcal{H}_{t}\right)$ in terms of the sup-norm of the initial data, and converges almost everywhere (when $h \rightarrow 0$ ) towards the unique entropy solution $u \in L_{l o c}^{\infty}\left(M_{+}\right)$to the Cauchy problem (2.1), (2.4). 
In section 5 below, we will derive the key estimates required for the proof of Theorem 3.3 which will be finally be given in section 6 . We follow here the strategy originally developed by Cockburn, Coquel and LeFloch [5, 7] for conservation laws posed on a fixed (time-independent) Euclidian background. New estimates are required here to take into account the geometric effects,especially those arising from the time evolution of the scheme. We will start with local (both in time and in space) entropy estimates, and next deduce a global-in-space entropy inequality. We will also establish the $L^{\infty}$ stability of the scheme and, finally, the global (spacetime) entropy inequality required for the convergence proof.

\section{Examples and remarks on our assumptions}

4.1. Admissible triangulations and lack of total variation estimate. Our assumption on the triangulation is essentially optimal. We argue by describing the setting in which the condition (3.15) will actually be used within our proof of Theorem 3.3. We also provide evidence that, in general, the finite volume method may not converge without this assumption. form

In the proof of convergence (see section 6), it is necessary to bound a term of the

$$
\begin{aligned}
A^{h}(X)=\sum_{K \in \mathcal{T}^{h}}( & \left|e_{K}^{+}\right| g\left(\mathbf{w}_{K}, X\left(p_{K}^{+}\right)\right) g\left(F\left(u_{K}^{-}, p_{K}^{+}\right), \mathbf{n}_{K, e_{K}^{+}}\right) \\
& \left.+\left|e_{K}^{-}\right| g\left(\mathbf{w}_{K^{-}}, X\left(p_{K}^{-}\right)\right) g\left(F\left(u_{K}^{-}, p_{K}^{-}\right), \mathbf{n}_{K, e_{K}^{-}}\right)\right),
\end{aligned}
$$

where $X$ is a smooth vector field, $p_{K}^{ \pm}$is the center of mass of $e_{K}^{ \pm}$, and the sum is taken over the whole spacetime triangulation. Recall that the vector $\mathbf{w}_{K}$ was defined earlier in this section. The above term must vanish in the limit for the finite volume schemes to converge and, furthermore, is an entirely new term that does not arise in the Euclidean nor Riemannian settings.

Note that both terms in the expression $A^{h}(X)$ involve $F\left(u_{K}^{-}, \cdot\right)$ and, consequently, the terms cannot be cancelled by re-ordering the expression. Therefore, if one were to integrate by parts the (discrete) sums, we would find ourselves in need of the uniform bound

$$
\sum_{K \in \mathcal{T}^{h}} h|| e_{K}^{+}\left|u_{K}^{+}-\right| e_{K}^{-}\left|u_{K}^{-}\right|=o(1) .
$$

However, it is well-known that this BV (bounded variation) time estimate is a very difficult open problem in the numerical analysis of finite volume schemes. Indeed, deriving (4.1) is open, even in the simplest Euclidean setting whenever the spatial discretization is not Cartesian.

On the other hand, one key observation made by Cockburn, Coquel, and LeFloch $[5,7]$ was that (4.1) was not necessary for the analysis of the convergence of the finite volume method, provided one considers $L^{\infty}$ solutions rather than solutions with bounded variation.

The notion of admissible triangulation introduced in the present paper supplements the observation in $[5,7]$ and provides the precise condition ensuring the convergence of the schemes. In the Euclidian or Riemannian cases, our admissibility condition imposes no new constraint on triangulations. In view of our condition in 
Definition 3.2 , it is easily checked that the term $A^{h}(X)$ converges to zero. Indeed, recalling the definition of $u^{h}$, we find

$$
\begin{aligned}
\left|A^{h}(X)\right| & =\left|\sum_{K \in \mathcal{T}^{h}}\left(|K| \mathcal{E}(K)-\left|K^{-}\right| \mathcal{E}\left(K^{-}\right)\right)\left(X, F\left(u^{h}\right)\right)\right| \\
& \lesssim \eta(h) \rightarrow 0 .
\end{aligned}
$$

Thus, no control on the total variation of the discrete solution is required. and instead the proposed geometric condition on the triangulation suffices. See section 4.4 for a further discussion.

4.2. Foliation by hypersurfaces and choice of triangulations. Our analysis is valid for any time-evolution that one may want to choose for the discretization, provided the assumptions on the triangulation in Definition 3.2 are met. These assumptions are independent of the actual foliation of the manifold appear to be essentially optimal, within the framework developed in the present paper. In fact, our method of proof is not tied to any particular time structure on the manifold - it only supposes that such a structure exists, which is a completely general assumption required for solving the initial value problem.

In particular, if a certain hypersurface $\mathcal{H}$ belongs to a given triangulation $\mathcal{T}^{h}$ (for some $h$ ), then this hypersurface need not be included in the triangulations $\mathcal{T}^{h^{\prime}}$ with $h^{\prime}<h$. That is to say, the discretization is not associated with any a priori fixed foliation, nor does the relation $\mathcal{T}^{h^{\prime}} \subset \mathcal{T}^{h}$ hold for $h^{\prime}<h$.

On the other hand, in Proposition 4.1 below, we are going to examine the special case where the triangulation is subordinate to a given foliation, and prove that it is admissible in the sense of Definition 3.2.

Furthermore, our formulas do coincide with the formulas already known in the Riemannian and Euclidean cases. In these cases, the function $\mu_{K, e_{K}^{-}}^{f}(u)$ coincides with the identity function $\mu_{K, e_{K}^{-}}^{f}(u)=u$ and, therefore, the finite volume scheme reduces to the scheme studied in $[1,5,7]$. Also, our expression for the time increment $\tau$ and the CFL condition (3.14) reduce to the usual formulas when specialized to the Euclidean or Riemannian setting.

4.3. Choice of flux-functions. Examples of scalar equations can be exhibited by taking any smooth, timelike vector field $X$ and any smooth real function $\widetilde{f}(u)$ and setting $f(u, p):=X(p) \widetilde{f}(u)$. The conservation law then reads $\operatorname{div}_{g}(X(p) \bar{f}(u(p)))=0$, and the flux is non-trivial and involves the geometry of the manifold.

In the interest of practical implementation, one may replace the right-hand side of the equations (3.3) and (3.6) with more realistic averages. For instance, one could take an average of $g\left(f(u, p), \mathbf{n}_{K, e}\right)$ over $N$ spatial points $p_{j}$ given from some partition $e^{j}$ of $e$,

$$
\mu_{K, e}^{f}(u)=\frac{1}{|e|} \sum_{j=1}^{N}\left|e^{j}\right| g\left(f\left(u, p_{j}\right), \mathbf{n}_{K, e}\left(p_{j}\right)\right) .
$$

Hence, more generally, one could fix an averaging operator $\mu_{K, e_{K}^{-}}^{f}$, and then use the equation (3.5) to iterate the method, with initial data given by

$$
u_{K}^{-}:=\mu_{e_{K}^{-}}^{u_{0}} .
$$


However, any such average is just an approximation of the integral expression used in (3.3). This approximation can be chosen to be of arbitrary high-order in the parameter $h$, by choosing appropriate quadrature formulas. For the sake of clarity, we will present the proofs with the choice $\mu_{K, e}^{f}$ defined by (3.3) and we will omit the (straightforward) treatment of the error terms issuing from the above approximations.

As an example of numerical flux, one can consider the following generalization of the Lax-Friedrichs flux,

$$
\mathbf{q}_{K, e^{0}}(u, v)=\frac{1}{2}\left(\mu_{K, e^{0}}^{f}(u)+\mu_{K, e^{0}}^{f}(v)\right)+\frac{D_{K, e^{0}}}{2}(u-v),
$$

where the constants $D_{K, e^{0}}$ satisfy $D_{K, e^{0}}=D_{K_{e} 0, e^{0}}$ and

$$
D_{K, e^{0}} \geq \frac{\left|e_{K}^{+}\right|}{\left|\partial^{0} K\right|}\left(\sup _{u \in \mathbb{R}} \partial_{u}\left(\mu_{K^{+}, e_{K}^{+}}^{f}\right)^{-1}(u)\right)^{-1} .
$$

This numerical flux is conservative and consistent, and it is monotone, as may be checked using the CFL condition (3.14).

4.4. A class of examples based on a geometric condition. We provide here an explicit condition which is geometric in nature and suffices for a triangulation to be admissible in the sense of (3.15). Recall that $p_{K}^{ \pm}$denotes the center of mass of $e_{K}^{ \pm}$and that the vector $\mathbf{w}_{K}$ denotes the tangent at $p_{K}^{+}$to the geodesic from $p_{K}^{+}$to the center of $\partial^{0} K$.

Proposition 4.1. Let $\mathcal{T}^{h}$ be a triangulation and suppose that, for each element $K$, the rescaled exterior normals $\left|e_{K}^{+}\right| \mathbf{n}_{K, e_{K}^{+}}$and $\left|e_{K}^{-}\right| \mathbf{n}_{K, e_{K}^{-}}$and the vectors $\mathbf{w}_{K}$ and $\mathbf{w}_{K^{-}}$ satisfy the following conditions: for every smooth vector field $X$,

$$
\begin{gathered}
\left|g\left(\left|e_{K}^{+}\right| \mathbf{n}_{K, e_{K}^{+}}, X\right)-g\left(\left|e_{K}^{-}\right| \mathbf{n}_{K, e_{K}^{-}}, X\right)\right| \lesssim \frac{\eta(h)}{h}|K|\|X\|_{L^{\infty}(K)}, \\
\left|g\left(\mathbf{w}_{K}, X\right)-g\left(\mathbf{w}_{K^{-}}, X\right)\right| \lesssim \eta(h) \tau_{K}\|X\|_{L^{\infty}(K)},
\end{gathered}
$$

where the expressions under consideration are evaluated at the centers of mass of $e_{K}^{+}$ and $e_{K}^{-}$, and $\eta(h)$ is such that $\eta(h) \rightarrow 0$. Then, $\mathcal{T}^{h}$ is an admissible triangulation in the sense of (3.15).

For instance, one can easily check that if a triangulation is subordinate to a given foliation (in the sense that the set of all outgoing elements $\left\{e_{K}^{+}: K \in \mathcal{T}^{h}\right\}$ is contained in a certain Cauchy surface), and if, moreover, each lateral element $e^{0}$ is everywhere tangent to a given, fixed, smooth timelike vector field, then the hypotheses of Proposition 4.1 hold. However, our condition (3.15) or the ones in Proposition 4.1 allow for more general triangulations, which need not satisfy such regularity assumptions.

Proof. Let $\Phi$ be a smooth vector field and, for each $K$, let $\Psi_{K}$ be a family of smooth vector fields defined on $\mathbf{M}$. We have

$$
\begin{aligned}
& \sum_{K \in \mathcal{T}^{h}}|K| \mathcal{E}(K)\left(\Phi, \Psi_{K}\right)-\left|K^{-}\right| \mathcal{E}\left(K^{-}\right)\left(\Phi, \Psi_{K}\right) \\
= & \sum_{K \in \mathcal{T}^{h}}\left(\left|e_{K}^{+}\right| \mathbf{w}_{K} \otimes \mathbf{n}_{K, e_{K}^{+}}-\left|e_{K}^{-}\right| \mathbf{w}_{K^{-}} \otimes \mathbf{n}_{K, e_{K}^{-}}\right)\left(\Phi, \Psi_{K}\right) \\
= & \sum_{K \in \mathcal{T}^{h}} g\left(\mathbf{w}_{K}, \Phi\right) g\left(\left|e_{K}^{+}\right| \mathbf{n}_{K, e_{K}^{+}}, \Psi_{K}\right)-g\left(\mathbf{w}_{K^{-}}, \Phi\right) g\left(\left|e_{K}^{-}\right| \mathbf{n}_{K, e_{K}^{-}}, \Psi_{K}\right),
\end{aligned}
$$


thus

$$
\begin{aligned}
& \quad\left|\sum_{K \in \mathcal{T}^{h}}\right| K\left|\mathcal{E}(K)\left(\Phi, \Psi_{K}\right)-\right| K^{-}\left|\mathcal{E}\left(K^{-}\right)\left(\Phi, \Psi_{K}\right)\right| \\
& =\mid \sum_{K \in \mathcal{T}^{h}} g\left(\mathbf{w}_{K^{-}}, \Phi\right)\left(g\left(\left|e_{K}^{+}\right| \mathbf{n}_{K, e_{K}^{+}}, \Psi_{K}\right)-g\left(\left|e_{K}^{-}\right| \mathbf{n}_{K, e_{K}^{-}}, \Psi_{K}\right)\right) \\
& \quad+\left(g\left(\mathbf{w}_{K}, \Phi\right)-g\left(\mathbf{w}_{K^{-}}, \Phi\right)\right) g\left(\left|e_{K}^{+}\right| \mathbf{n}_{K, e_{K}^{+}}, \Psi_{K}\right) \mid \\
& \quad \eta(h) \sum_{K \in \mathcal{T}^{h}}|K|\|\Phi\|_{L^{\infty}}\left\|\Psi_{K}\right\|_{L^{\infty}} \lesssim \eta(h)\|\Phi\|_{L^{\infty} \sup _{K}}\left\|\Psi_{K}\right\|_{L^{\infty}} .
\end{aligned}
$$

In view of (3.15), this shows that $\mathcal{T}^{h}$ is an admissible triangulation.

\section{Discrete entropy estimates}

5.1. Local entropy dissipation and entropy inequalities. We now introduce some notation which will simplify the statement of the results as well as the proofs. By defining

$$
\begin{aligned}
\mu_{K}^{+}(u):= & \mu_{K^{+}, e_{K}^{+}}^{f}(u)=-\mu_{K, e_{K}^{+}}^{f}(u), \\
& \mu_{K}^{-}(u):=\mu_{K, e_{K}^{-}}^{f},
\end{aligned}
$$

the finite volume method (3.5) reads as

$$
\left|e_{K}^{+}\right| \mu_{K}^{+}\left(u_{K}^{+}\right)=\left|e_{K}^{-}\right| \mu_{K}^{-}\left(u_{K}^{-}\right)-\sum_{e^{0} \in \partial^{0} K}\left|e^{0}\right| \mathbf{q}_{K, e^{0}}\left(u_{K}^{-}, u_{K_{e^{0}}}^{-}\right) .
$$

As in $[1,5,7]$, we rely on a convex decomposition of $\mu_{K}^{+}\left(u_{K}^{+}\right)$, which allows us to control the entropy dissipation.

Define $\tilde{\mu}_{K, e^{0}}^{+}$by the identity

$$
\tilde{\mu}_{K, e^{0}}^{+}:=\mu_{K}^{+}\left(u_{K}^{-}\right)-\frac{\left|\partial^{0} K\right|}{\left|e_{K}^{+}\right|}\left(\mathbf{q}_{K, e^{0}}\left(u_{K}^{-}, u_{K_{e^{0}}}^{-}\right)-\mathbf{q}_{K, e^{0}}\left(u_{K}^{-}, u_{K}^{-}\right)\right),
$$

and define

$$
\bar{\mu}_{K, e^{0}}^{+}:=\tilde{\mu}_{K, e^{0}}^{+}-\frac{1}{\left|e_{K}^{+}\right|} \int_{K} \operatorname{div}_{g} f\left(u_{K}^{-}, p\right) d V_{K} .
$$

Then, one has the following convex decomposition of $\mu_{K}^{+}\left(u_{K}^{+}\right)$, whose proof is immediate from (5.1).

$$
\mu_{K}^{+}\left(u_{K}^{+}\right)=\frac{1}{\left|\partial^{0} K\right|} \sum_{e^{0} \in \partial^{0} K}\left|e^{0}\right| \bar{\mu}_{K, e^{0}}^{+} .
$$

Lemma 5.1. Let $(U(u), F(u, p))$ be a convex entropy pair (cf. Definition 2.1). For each $K$ and for each $e=e_{K}^{-}, e_{K}^{+}$, let $V_{K, e}: \mathbb{R} \rightarrow \mathbb{R}$ be the convex function defined by

$$
V_{K, e}(a):=\mu_{K, e}^{F}\left(\left(\mu_{K, e}^{f}\right)^{-1}(a)\right), \quad a \in \mathbb{R} .
$$

Then there exists a family of numerical entropy fluxes $\mathbf{Q}_{K, e^{0}}(u, v): \mathbb{R}^{2} \rightarrow \mathbb{R}$ satisfying the following conditions. 
- $\mathbf{Q}_{K, e^{0}}$ is consistent with the entropy flux $F$ :

$$
\mathbf{Q}_{K, e^{0}}(u, u)=\mu_{K, e^{0}}^{F}(u), \quad K \in \mathcal{T}^{h}, e^{0} \in \partial^{0} K, u \in \mathbb{R} .
$$

- Conservation property:

$$
\mathbf{Q}_{K, e^{0}}(u, v)=-\mathbf{Q}_{K_{e^{0}}, e^{0}}(v, u), \quad u, v \in \mathbb{R} .
$$

- Discrete entropy inequality:

$$
\begin{aligned}
& V_{K^{+}, e_{K}^{+}}\left(\tilde{\mu}_{K, e^{0}}^{+}\right)-V_{K^{+}, e_{K}^{+}}\left(\mu_{K}^{+}\left(u_{K}^{-}\right)\right) \\
& \quad+\frac{\left|\partial^{0} K\right|}{\left|e_{K}^{+}\right|}\left(\mathbf{Q}_{K, e^{0}}\left(u_{K}^{-}, u_{K_{e}^{0}}^{-}\right)-\mathbf{Q}_{K, e^{0}}\left(u_{K}^{-}, u_{K}^{-}\right)\right) \leq 0 .
\end{aligned}
$$

From the inequality (5.5) we infer that

$$
\begin{aligned}
& V_{K^{+}, e_{K}^{+}}\left(\bar{\mu}_{K, e^{0}}^{+}\right)-V_{K^{+}, e_{K}^{+}}\left(\mu_{K}^{+}\left(u_{K}^{-}\right)\right) \\
& \quad+\frac{\left|\partial^{0} K\right|}{\left|e_{K}^{+}\right|}\left(\mathbf{Q}_{K, e^{0}}\left(u_{K}^{-}, u_{K_{e^{0}}}^{-}\right)-\mathbf{Q}_{K, e^{0}}\left(u_{K}^{-}, u_{K}^{-}\right)\right) \leq R_{K, e^{0}}^{+},
\end{aligned}
$$

where $R_{K, e^{0}}^{+}$is given by

$$
R_{K, e^{0}}^{+}:=V_{K^{+}, e_{K}^{+}}\left(\bar{\mu}_{K, e^{0}}^{+}\right)-V_{K^{+}, e_{K}^{+}}\left(\tilde{\mu}_{K, e^{0}}^{+}\right) .
$$

Proof. To begin, we prove that the functions $V_{K, e}$ in (5.4) are indeed convex. First, note that it is sufficient to show that

$$
V_{K, e}(\mu)=\int^{\mu} U^{\prime}\left(\left(\mu_{K, e}^{f}\right)^{-1}(\sigma)\right) d \sigma
$$

Indeed, using the convexity of $U$ and the monotonicity of $\left(\mu_{K, e}^{f}\right)^{-1}$, for $e=e_{K}^{+}, e_{K}^{-}$(cf. Lemma 3.1), the convexity of $V_{K, e}$ follows by differentiating this expression twice. To prove (5.8), note that setting $\alpha=\left(\mu_{K, e}^{f}\right)^{-1}(\sigma)$, we find

$$
\begin{aligned}
\int^{\mu} U^{\prime}\left(\left(\mu_{K, e}^{f}\right)^{-1}(\sigma)\right) d \sigma & =\int^{\left(\mu_{K, e}^{f}\right)^{-1}(\mu)} U^{\prime}(\alpha) \partial_{\alpha} \mu_{K, e}^{f}(\alpha) d \alpha \\
& =\oint_{e} g\left(\int^{\left(\mu_{K, e}^{f}\right)^{-1}(\mu)} U^{\prime}(\alpha) \partial_{\alpha} f(\alpha, p) d \alpha, \mathbf{n}_{K, e}(p)\right) d V_{e} \\
& =\oint_{e} g\left(F\left(\left(\mu_{K, e}^{f}\right)^{-1}(\mu), p\right), \mathbf{n}_{K, e}(p)\right) d V_{e}=\mu_{K, e}^{F}\left(\left(\mu_{K, e}^{f}\right)^{-1}(\mu)\right),
\end{aligned}
$$

which establishes (5.8).

We now proceed with the proof of the lemma. First of all, note that using (5.4) we may write the inequality (5.5) equivalently as

$$
\begin{aligned}
& \mu_{K^{+}, e_{K}^{+}}^{F}\left(\left(\mu_{K^{+}, e_{K}^{+}}^{f}\right)^{-1}\left(\tilde{\mu}_{K, e^{0}}^{+}\right)\right)-\mu_{K^{+}, e_{K}^{+}}^{F}\left(u_{K}^{-}\right) \\
& \quad+\frac{\left|\partial^{0} K\right|}{\left|e_{K}^{+}\right|}\left(\mathbf{Q}_{K, e^{0}}\left(u_{K}^{-}, u_{K_{e^{0}}}^{-}\right)-\mathbf{Q}_{K, e^{0}}\left(u_{K}^{-}, u_{K}^{-}\right)\right) \leq 0 .
\end{aligned}
$$


Indeed, we have for instance

$$
V_{K^{+}, e_{K}^{+}}\left(\mu_{K}^{+}\left(u_{K}^{-}\right)\right)=\mu_{K^{+}, e_{K}^{+}}^{F}\left(\left(\mu_{K^{+}, e_{K}^{+}}^{f}\right)^{-1}\left(\mu_{K}^{+}\left(u_{K}^{-}\right)\right)\right)=\mu_{K^{+}, e_{K}^{+}}^{F}\left(u_{K}^{-}\right) .
$$

Next, introduce the following operator. For $u, v \in \mathbb{R}, e^{0} \in \partial^{0} K$, let

$$
H_{K, e^{0}}(u, v):=\mu_{K}^{+}(u)-\frac{\left|\partial^{0} K\right|}{\left|e_{K}^{+}\right|}\left(\mathbf{q}_{K, e^{0}}(u, v)-\mathbf{q}_{K, e^{0}}(u, u)\right) .
$$

We claim that $H_{K, e^{0}}$ satisfies the following properties:

$$
\begin{gathered}
\frac{\partial}{\partial u} H_{K, e^{0}}(u, v) \geq 0, \quad \frac{\partial}{\partial v} H_{K, e^{0}}(u, v) \geq 0 \\
H_{K, e^{0}}(u, u)=\mu_{K}^{+}(u) .
\end{gathered}
$$

The second and last properties are immediate. The first is a consequence of the CFL condition (3.14) and the monotonicity of the method. Indeed, from the definition of $H_{K, e^{0}}(u, v)$ we may perform exactly the same calculation as in the proof of Lemma 5.2 to prove that $H_{K, e^{0}}(u, v)$ is a convex combination of $\mu_{K}^{+}(u)$ and $\mu_{K}^{+}(v)$, which in turn are increasing functions. This establishes the first inequality in (5.10).

We now turn to the proof of the entropy inequality (5.9). Suppose first that (5.9) is already established for the Kruzkov family of entropies $\bar{U}(u, \lambda)=|u-\lambda|, \bar{F}(u, \lambda, p)=$ $\operatorname{sgn}(u-\lambda)(f(u, p)-f(\lambda, p)), \lambda \in \mathbb{R}$. In this case, the Kruzkov numerical entropy fluxes are given by

$$
\overline{\mathbf{Q}}_{K, e^{0}}(u, v, \lambda):=\mathbf{q}_{K, e^{0}}(u \vee \lambda, v \vee \lambda)-\mathbf{q}_{K, e^{0}}(u \wedge \lambda, v \wedge \lambda)
$$

where $a \vee b=\max (a, b)$, and $a \wedge b=\min (a, b)$. It is easy to check that $\mathbf{Q}_{K, e^{0}}$ satisfies the first two conditions of the lemma.

We now show that it is enough to prove inequality (5.9) for Kruzkov's entropies only. Indeed, if $U$ is a smooth function which is linear at infinity, we have (formally)

$$
\begin{aligned}
\frac{1}{2} \int_{\mathbb{R}} \bar{U}(u, \lambda) U^{\prime \prime}(\lambda) d \lambda & =\frac{1}{2} \int_{\mathbb{R}} \bar{U}^{\prime \prime}(u, \lambda) U(\lambda) d \lambda \\
& =\frac{1}{2}\left\langle\delta_{\lambda=u}, U(\lambda)\right\rangle=U(u),
\end{aligned}
$$

modulo an additive constant. Similarly, if $(U, F)$ is a convex entropy pair, we obtain

$$
\frac{1}{2} \int_{\mathbb{R}} \bar{F}(u, \lambda, p) U^{\prime \prime}(\lambda) d \lambda=F(u, p) .
$$

Since we shall prove an $L^{\infty}$ bound for our approximate solutions, we may suppose that the $u$ above varies in a bounded set $B \subset \mathbb{R}$. Thus, we may apply the same reasoning with any function which is not linear at infinity, by changing it into a linear function outside $B$. This shows that we can obtain the inequality (5.9) for any convex entropy pair $(U, F)$ by first proving it in the special case of Kruzkov's entropies, multiplying by $U^{\prime \prime}(\lambda) / 2$, and integrating. In that case, the numerical flux will be given by

$$
\mathbf{Q}_{K, e^{0}}(u, v)=\frac{1}{2} \int_{\mathbb{R}} \overline{\mathbf{Q}}_{K, e^{0}}(u, v, \lambda) U^{\prime \prime}(\lambda) d \lambda .
$$


Again, this numerical flux satisfies the first two assumptions of the lemma, since they are inherited from the corresponding properties for the Kruzkov numerical flux $\overline{\mathbf{Q}}_{K, e^{0}}(u, v, \lambda)$.

Therefore, we now proceed to prove the inequality (5.9) for Kruzkov's family of entropies. This is done in two steps. First, we will show that

$$
\begin{aligned}
\mu_{K^{+}, e_{K}^{+}}^{\bar{F}}\left(u_{K}^{-}, \lambda\right) & -\frac{\left|\partial^{0} K\right|}{\left|e_{K}^{+}\right|}\left(\overline{\mathbf{Q}}_{K, e^{0}}\left(u_{K}^{-}, u_{K_{e^{0}}}^{-}, \lambda\right)-\overline{\mathbf{Q}}_{K, e^{0}}\left(u_{K}^{-}, u_{K}^{-}, \lambda\right)\right) \\
& =H\left(u_{K}^{-} \vee \lambda, u_{K_{e^{0}}}^{-} \vee \lambda\right)-H\left(u_{K}^{-} \wedge \lambda, u_{K_{e^{0}}}^{-} \wedge \lambda\right) .
\end{aligned}
$$

Second, we will see that for any $u, v, \lambda \in \mathbb{R}$, we have

$$
H(u \vee \lambda, v \vee \lambda)-H(u \wedge \lambda, v \wedge \lambda) \geq \mu_{K^{+}, e_{K}^{+}}^{\bar{F}}\left(\left(\mu_{K}^{+}\right)^{-1}(H(u, v)), \lambda\right) .
$$

For ease of notation, we omit $K, e^{0}$ from the expression of $H$. The identity (5.12) and the inequality (5.13) (with $u=u_{K}^{-}, v=u_{K_{e^{0}}}^{-}$) combined give (5.9), for Kruzkov's entropies.

To prove (5.12), simply observe that

$$
\begin{aligned}
\mu_{K^{+}, e_{K}^{+}}^{\bar{F}}\left(u_{K}^{-}, \lambda\right) & =\operatorname{sgn}\left(u_{K}^{-}-\lambda\right)\left(\mu_{K^{+}, e_{K}^{+}}^{f}\left(u_{K}^{-}\right)-\mu_{K^{+}, e_{K}^{+}}^{f}(\lambda)\right) \\
& =\operatorname{sgn}\left(\mu_{K}^{+}\left(u_{K}^{-}\right)-\mu_{K}^{+}(\lambda)\right)\left(\mu_{K}^{+}\left(u_{K}^{-}\right)-\mu_{K}^{+}(\lambda)\right) \\
& =\left(\mu_{K}^{+}\left(u_{K}^{-}\right) \vee \mu_{K}^{+}(\lambda)-\mu_{K}^{+}\left(u_{K}^{-}\right) \wedge \mu_{K}^{+}(\lambda)\right) \\
& =\left(\mu_{K}^{+}\left(u_{K}^{-} \vee \lambda\right)-\mu_{K}^{+}\left(u_{K}^{-} \wedge \lambda\right)\right) .
\end{aligned}
$$

Here, we have repeatedly used that $\mu_{K}^{+}$is a monotone increasing function. The identity (5.12) now follows from the expressions of the Kruzkov numerical entropy flux, $\overline{\mathbf{Q}}_{K, e^{0}}$, and of $H$.

Consider now the inequality (5.13). We have

$$
\begin{aligned}
H(u \vee \lambda, v \vee \lambda) & -H(u \wedge \lambda, v \wedge \lambda) \\
& \geq(H(u, v) \vee H(\lambda, \lambda))-(H(u, v) \wedge H(\lambda, \lambda)) .
\end{aligned}
$$

This is a consequence of the fact that if $\varphi$ is an increasing function, then $\varphi(u \vee \lambda)=$ $\varphi(u \vee \lambda) \vee \varphi(u \vee \lambda) \geq \varphi(u) \vee \varphi(\lambda)$, and (5.10). Thus, we have

$$
\begin{aligned}
& H(u \vee \lambda, v \vee \lambda)-H(u \wedge \lambda, v \wedge \lambda) \\
\geq & |H(u, v)-H(\lambda, \lambda)|=\left|H(u, v)-\mu_{K}^{+}(\lambda)\right| \\
= & \operatorname{sgn}\left(H(u, v)-\mu_{K}^{+}(\lambda)\right)\left(H(u, v)-\mu_{K}^{+}(\lambda)\right) \\
= & \operatorname{sgn}\left(\left(\mu_{K}^{+}\right)^{-1}(H(u, v))-\lambda\right)\left(\mu_{K}^{+}\left(\left(\mu_{K}^{+}\right)^{-1}(H(u, v))\right)-\mu_{K}^{+}(\lambda)\right) \\
= & \mu_{K^{+}, e_{K}^{+}}^{\bar{F}}\left(\left(\mu_{K}^{+}\right)^{-1}(H(u, v)), \lambda\right) .
\end{aligned}
$$

This establishes (5.13). We now choose $u=u_{K}^{-}, v=u_{K_{e^{0}}}^{-}$in (5.13), observe that $H_{K, e^{0}}\left(u_{K}^{-}, u_{K_{e^{0}}}^{-}\right)=\tilde{\mu}_{K, e^{0}}^{+}$, and combine this with (5.12) to obtain inequality (5.9) for the Kruzkov entropies. As described above, (5.9) will hold for all convex entropy pairs $(U, F)$. This completes the proof of Lemma 5.1. 
5.2. Entropy dissipation estimate and $L^{\infty}$ estimate. We now discuss the time evolution of the triangulation. As we have said, the initial hypersurface $\mathcal{H}_{0}$ is composed of inflow elements $e_{K}^{-}$. We then define the hypersurfaces $\mathcal{H}_{n}$, for $n>0$, by

$$
\mathcal{H}_{n}:=\bigcup_{e_{K}^{-} \subset \mathcal{H}_{n-1}} e_{K}^{+}
$$

and set

$$
\mathcal{K}^{n}:=\left\{K: e_{K}^{-} \subset \mathcal{H}_{n-1}, \quad e_{K}^{+} \subset \mathcal{H}_{n}\right\} .
$$

It is important to note that the hypersurfaces $\mathcal{H}_{n}$ are not necessarily associated with a foliation $\left\{\mathcal{H}_{t}\right\}$ of the manifold; they are only restricted by our admissibility assumptions in Definition 3.2.

Next, we introduce the following notation, which we will use from now on. For $K \in \mathcal{K}^{n}$, we write

$$
\mu_{K}^{n}:=\mu_{K}^{-}=\mu_{K, e_{K}^{-}}^{f}, \quad u_{K}^{n}:=u_{K}^{-}, \quad \bar{\mu}_{K, e^{0}}^{n+1}:=\bar{\mu}_{K, e^{0}}^{+},
$$

so that, for instance, $\mu_{K}^{+}\left(u_{K}^{+}\right)=\mu_{K}^{n+1}\left(u_{K}^{n+1}\right)$. Accordingly, we define

$$
V_{K}^{n}(\mu):=V_{K, e_{K}^{-}}(\mu), \quad R_{K, e^{0}}^{n+1}:=R_{K, e^{0}}^{+},
$$

where the timelike entropy flux $V_{K, e}$ and the error term $R_{K, e^{0}}^{+}$are defined in Lemma 5.1 .

Lemma 5.2. The finite volume approximations satisfy the $L^{\infty}$ bound

$$
\max _{K \in \mathcal{K}^{n}}\left|u_{K}^{n}\right| \leq\left(\max _{K^{0} \in \mathcal{K}^{0}}\left|u_{K}^{0}\right|+C_{1} t_{n}\right) e^{C_{2} t_{n}}
$$

for some constants $C_{1}, C_{2} \geq 0$, where

$$
t_{n}:=\sum_{j=0}^{n} \tau_{j}=\sum_{j=0}^{n} \max _{K^{j} \in \mathcal{K}_{j}} \frac{\left|K^{j}\right|}{\left|e_{K^{j}}^{+}\right|} .
$$

Proof. First of all, observe that from the consistency condition (3.9), the definition of $\mu_{K, e}^{f}$ in (3.3) and the divergence theorem, we have for any $u \in \mathbb{R}$,

$$
\begin{aligned}
\int_{K} \operatorname{div}_{g} f(u, p) d V_{K} & =\int_{\partial K} g_{p}(f(u, p), \tilde{\mathbf{n}}(p)) d V_{\partial K} \\
& =\left|e_{K}^{+}\right| \mu_{K}^{n+1}(u)-\left|e_{K}^{-}\right| \mu_{K}^{n}(u)+\sum_{e^{0} \in \partial^{0} K}\left|e^{0}\right| \mathbf{q}_{K, e^{0}}(u, u)
\end{aligned}
$$

(recall that $\tilde{\mathbf{n}}$ is the interior unit normal if it is timelike, and the exterior unit normal if it is spacelike). Moreover, with our notation the finite volume scheme (3.4) reads as

$$
\left|e_{K}^{+}\right| \mu_{K}^{n+1}\left(u_{K}^{n+1}\right)=\left|e_{K}^{-}\right| \mu_{K}^{n}\left(u_{K}^{n}\right)-\sum_{e^{0} \in \partial^{0} K}\left|e^{0}\right| \mathbf{q}_{K, e^{0}}\left(u_{K}^{n}, u_{K_{e^{0}}}^{n}\right) .
$$


Combining these two identities gives

$$
\begin{aligned}
\mu_{K}^{n+1}\left(u_{K}^{n+1}\right)= & \mu_{K}^{n+1}\left(u_{K}^{n}\right)-\frac{1}{\left|e_{K}^{+}\right|} \int_{K} \operatorname{div}_{g} f\left(u_{K}^{n}, p\right) d V_{K} \\
& -\sum_{e^{0} \in \partial^{0} K} \frac{\left|e^{0}\right|}{\left|e_{K}^{+}\right|}\left(\mathbf{q}_{K, e^{0}}\left(u_{K}^{n}, u_{K_{e^{0}}}^{n}\right)-\mathbf{q}_{K, e^{0}}\left(u_{K}^{n}, u_{K}^{n}\right)\right) .
\end{aligned}
$$

Next, we rewrite the right-hand side as follows:

$$
\begin{aligned}
\mu_{K}^{n+1}\left(u_{K}^{n+1}\right)= & \left(1-\sum_{e^{0} \in \partial^{0} K} \alpha_{K, e^{0}}\right) \mu_{K}^{n+1}\left(u_{K}^{n}\right)+\sum_{e^{0} \in \partial^{0} K} \alpha_{K, e^{0}} \mu_{K}^{n+1}\left(u_{K_{e^{0}}}^{n}\right) \\
& -\frac{1}{\left|e_{K}^{+}\right|} \int_{K} \operatorname{div}_{g} f\left(u_{K}^{n}, p\right) d V_{K}
\end{aligned}
$$

where

$$
\alpha_{K, e^{0}}:=\frac{\left|e^{0}\right|}{\left|e_{K}^{+}\right|} \frac{\mathbf{q}_{K, e^{0}}\left(u_{K}^{n}, u_{K_{e} 0}^{n}\right)-\mathbf{q}_{K, e^{0}}\left(u_{K}^{n}, u_{K}^{n}\right)}{\mu_{K}^{n+1}\left(u_{K}^{n}\right)-\mu_{K}^{n+1}\left(u_{K^{0}}^{n}\right)} .
$$

This gives a convex combination of $\mu_{K}^{n+1}\left(u_{K}^{n}\right)$ and $\mu_{K}^{n+1}\left(u_{K_{e} 0}^{n}\right)$. Indeed, on one hand we have $\sum_{e^{0} \in \partial^{0} K} \alpha_{K, e^{0}} \geq 0$, due to the monotonicity condition (3.11) and Lemma 3.1. On the other hand, the CFL condition (3.14) gives us

$$
\begin{aligned}
\sum_{e^{0} \in \partial^{0} K} \alpha_{K, e^{0}} & <\left|\frac{u_{K}^{n}-u_{K_{e^{0}}}^{n}}{\mu_{K}^{n+1}\left(u_{K}^{n}\right)-\mu_{K}^{n+1}\left(u_{K_{e} 0}^{n}\right)}\right|\left(\operatorname{Lip}\left(\mu_{K}^{n+1}\right)^{-1}\right)^{-1} \\
& \leq \operatorname{Lip}\left(\mu_{K}^{n+1}\right)^{-1} / \operatorname{Lip}\left(\mu_{K}^{n+1}\right)^{-1}=1 .
\end{aligned}
$$

Thus, we find

$$
\begin{aligned}
& \mu_{K}^{n+1}\left(u_{K}^{n+1}\right) \geq \min \left(\mu_{K}^{n+1}\left(u_{K}^{n}\right), \min _{e^{0} \in \partial^{0} K} \mu_{K}^{n+1}\left(u_{K_{e^{0}}}^{n}\right)\right)-\frac{1}{\left|e_{K}^{+}\right|} \int_{K} \operatorname{div}_{g} f(u, p) d V_{K}, \\
& \mu_{K}^{n+1}\left(u_{K}^{n+1}\right) \leq \max \left(\mu_{K}^{n+1}\left(u_{K}^{n}\right), \max _{e^{0} \in \partial^{0} K} \mu_{K}^{n+1}\left(u_{K_{e^{0}}}^{n}\right)\right)-\frac{1}{\left|e_{K}^{+}\right|} \int_{K} \operatorname{div}_{g} f(u, p) d V_{K} .
\end{aligned}
$$

Composing with the monotone increasing function $\left(\mu_{K}^{n+1}\right)^{-1}$, we find

$$
\begin{aligned}
& u_{K}^{n+1} \geq \min \left(u_{K}^{n}, \min _{e^{0} \in \partial^{0} K} u_{K_{e^{0}}}^{n}\right)+\frac{\operatorname{Lip}\left(\mu_{K}^{n+1}\right)^{-1}}{\left|e_{K}^{+}\right|} \int_{K}\left|\operatorname{div}_{g} f\left(u_{K}^{n}, p\right)\right| d V_{K}, \\
& u_{K}^{n+1} \leq \max \left(u_{K}^{n}, \max _{e^{0} \in \partial^{0} K} u_{K_{e^{0}}}^{n}\right)+\frac{\operatorname{Lip}\left(\mu_{K}^{n+1}\right)^{-1}}{\left|e_{K}^{+}\right|} \int_{K}\left|\operatorname{div}_{g} f\left(u_{K}^{n}, p\right)\right| d V_{K},
\end{aligned}
$$

which in turn gives

$$
\left|u_{K}^{n+1}\right| \leq \max _{K \in \mathcal{K}^{n}}\left|u_{K}^{n}\right|+\max _{K \in \mathcal{K}^{n}} \frac{\operatorname{Lip}\left(\mu_{K}^{n+1}\right)^{-1}}{\left|e_{K}^{+}\right|} \int_{K}\left|\operatorname{div}_{g} f\left(u_{K}^{n}, p\right)\right| d V_{K} .
$$

By induction we obtain

$$
\left|u_{K}^{n+1}\right| \leq \max _{K^{0} \in \mathcal{K}^{0}}\left|u_{K}^{0}\right|+\sum_{j=0}^{n} \max _{K^{j} \in \mathcal{K}^{j}} \frac{\operatorname{Lip}\left(\mu_{K}^{j+1}\right)^{-1}}{\left|e_{K^{j}}^{+}\right|} \int_{K^{j}}\left|\operatorname{div}_{g} f\left(u_{K}^{j}, p\right)\right| d V_{K} .
$$


Now, we use the growth condition (2.5) on the last term,

$$
\begin{aligned}
& \sum_{j=0}^{n} \max _{K^{j} \in \mathcal{K}^{j}} \frac{\operatorname{Lip}\left(\mu_{K}^{j+1}\right)^{-1}}{\left|e_{K^{j}}^{+}\right|} \int_{K^{j}}\left|\operatorname{div}_{g} f\left(u_{K}^{j}, p\right)\right| d V_{K} \\
& \leq \sum_{j=0}^{n} \max _{K^{j} \in \mathcal{K}^{j}} \frac{\operatorname{Lip}\left(\mu_{K}^{j+1}\right)^{-1}}{\left|e_{K^{j}}^{+}\right|}\left|K^{j}\right|\left(C_{1}+C_{2}\left|u_{K}^{j}\right|\right) \\
& \leq\left(C_{1} t_{n}+C_{2} \sum_{j=0}^{n} \tau^{j} \max _{j}\left|u_{K}^{j}\right|\right) .
\end{aligned}
$$

Here, the constants $C_{1,2}$ may change at each occurrence, and we also used the fact that

$$
\max _{K^{j} \in \mathcal{K}^{j}} \operatorname{Lip}\left(\mu_{K}^{j+1}\right)^{-1} \leq C,
$$

which is an easy consequence of our assumptions on the flux $f$. The result now follows from a discrete version of the Gronwall inequality (see [1, Lemma 6.1]). This completes the proof of Lemma 5.2.

Recall that if $V$ is a convex function, then its modulus of convexity on a set $S$ is defined by $\beta:=\inf \left\{V^{\prime \prime}(w): w \in S\right\}$.

Proposition 5.3. Let $V_{K}^{n}$ be defined by (5.4), (5.14), and let $\beta_{K}^{n}$ be the modulus of convexity of $V_{K}^{n}$. Then, one has

$$
\begin{aligned}
& \sum_{K \in \mathcal{K}^{n}}\left|e_{K}^{+}\right| V_{K}^{n+1}\left(\mu_{K}^{n+1}\left(u_{K}^{n+1}\right)\right) \\
& \quad+\sum_{\substack{K \in \mathcal{K}^{n} \\
e^{0} \in \partial^{0} K}} \frac{\beta_{K}^{n+1}}{2} \frac{\left|e^{0}\right|\left|e_{K}^{+}\right|}{\left|\partial^{0} K\right|}\left|\bar{\mu}_{K, e^{0}}^{n+1}-\mu_{K}^{n+1}\left(u_{K}^{n+1}\right)\right|^{2} \\
& \leq \sum_{K \in \mathcal{K}^{n}}\left|e_{K}^{-}\right| V_{K}^{n}\left(\mu_{K}^{n}\left(u_{K}^{n}\right)\right) \\
& \quad+\sum_{K \in \mathcal{K}^{n}} \int_{K} \operatorname{div}_{g} F\left(u_{K}^{n}, p\right) d V_{K}+\sum_{\substack{K \in \mathcal{K}^{n} \\
e^{0} \in \partial^{0} K}} \frac{\left|e^{0}\right|\left|e_{K}^{+}\right|}{\left|\partial^{0} K\right|} R_{K, e^{0}}^{n+1} .
\end{aligned}
$$

Proof. Consider the discrete entropy inequality (5.6). Multiplying by $\frac{\left|e^{0}\right|\left|e_{K}^{+}\right|}{\left|\partial^{0} K\right|}$ and summing in $K \in \mathcal{K}^{n}, e^{0} \in \partial^{0} K$ gives

$$
\begin{aligned}
& \sum_{\substack{K \in \mathcal{K}^{n} \\
e^{0} \in \partial^{0} K}} \frac{\left|e^{0}\right|\left|e_{K}^{+}\right|}{\left|\partial^{0} K\right|} V_{K}^{n+1}\left(\bar{\mu}_{K, e^{0}}^{n+1}\right)-\sum_{K \in \mathcal{K}^{n}}\left|e_{K}^{+}\right| V_{K}^{n+1}\left(\mu_{K}^{n+1}\left(u_{K}^{n}\right)\right) \\
& +\sum_{\substack{K \in \mathcal{K}^{n} \\
e^{0} \in \partial^{0} K}}\left|e^{0}\right|\left(\mathbf{Q}_{K, e^{0}}\left(u_{K}^{n}, u_{K_{e^{0}}}^{n}\right)-\mathbf{Q}_{K, e^{0}}\left(u_{K}^{n}, u_{K}^{n}\right)\right) \\
\leq & \sum_{\substack{K \in \mathcal{K}^{n} \\
e^{0} \in \partial^{0} K}} \frac{\left|e^{0}\right|\left|e_{K}^{+}\right|}{\left|\partial^{0} K\right|} R_{K, e^{0}}^{n+1} .
\end{aligned}
$$


Next, observe that the conservation property (3.10) gives

$$
\sum_{\substack{K \in \mathcal{K}^{n} \\ e^{0} \in \partial^{0} K}}\left|e^{0}\right| \mathbf{Q}_{K, e^{0}}\left(u_{K}^{n}, u_{K_{e^{0}}}^{n}\right)=0 .
$$

Now, if $V$ is a convex function, and if $v=\sum_{j} \alpha_{j} v_{j}$ is a convex combination of $v_{j}$, then an elementary result on convex functions gives

$$
V(v)+\frac{\beta}{2} \sum_{j} \alpha_{j}\left|v_{j}-v\right|^{2} \leq \sum_{j} \alpha_{j} V\left(v_{j}\right) .
$$

Now, apply this result with the convex combination (5.3) and with the convex function $V_{K}^{n+1}$, multiply by $\left|e_{K}^{+}\right|$, and sum up in $K \in \mathcal{K}^{n}$. Then, combining the resulting inequality with (5.20), (5.21), we obtain

$$
\begin{aligned}
& \sum_{K \in \mathcal{K}^{n}}\left|e_{K}^{+}\right| V_{K}^{n+1}\left(\mu_{K}^{n+1}\left(u_{K}^{n+1}\right)\right)-\sum_{K \in \mathcal{K}^{n}}\left|e_{K}^{+}\right| V_{K}^{n+1}\left(\mu_{K}^{n+1}\left(u_{K}^{n}\right)\right) \\
& +\sum_{\substack{K \in \mathcal{K}^{n} \\
e^{0} \in \partial^{0} K}} \frac{\beta_{K}^{n+1}}{2} \frac{\left|e^{0}\right|\left|e_{K}^{+}\right|}{\left|\partial^{0} K\right|}\left|\bar{\mu}_{K, e^{0}}^{n+1}-\mu_{K}^{n+1}\left(u_{K}^{n+1}\right)\right|^{2} \\
& -\sum_{\substack{K \in \mathcal{K}^{n} \\
e^{0} \in \partial^{0} K}}\left|e^{0}\right| \mathbf{Q}_{K, e^{0}}\left(u_{K}^{n}, u_{K}^{n}\right) \leq \sum_{\substack{K \in \mathcal{K}^{n} \\
e^{0} \in \partial^{0} K}} \frac{\left|e^{0}\right|\left|e_{K}^{+}\right|}{\left|\partial^{0} K\right|} R_{K, e^{0}}^{n+1} .
\end{aligned}
$$

Finally, using the identity

$$
\begin{aligned}
& \int_{K} \operatorname{div}_{g} F(u, p) d V_{K}=\int_{\partial K} g_{p}(F(u, p), \tilde{\mathbf{n}}(p)) d V_{\partial K} \\
= & \left|e_{K}^{+}\right| V_{K}^{n+1}\left(\mu_{K}^{n+1}(u)\right)-\left|e_{K}^{-}\right| V_{K}^{n}\left(\mu_{K}^{n}(u)\right)+\sum_{e^{0} \in \partial^{0} K}\left|e^{0}\right| \mathbf{Q}_{K, e^{0}}(u, u)
\end{aligned}
$$

(with $u=u_{K}^{n}$ ) yields the desired result. This completes the proof of Proposition 5.3. COROLLARY 5.4. Suppose that for each $K \in \mathcal{T}^{h}, e=e_{K}^{ \pm}$, the function $V_{K, e}$ is strictly convex, and that, moreover, one has

$$
\beta_{K}^{n} \geq \beta>0,
$$

uniformly in $K$ and $n$. Then one has the following global estimate for the entropy dissipation,

$$
\sum_{n=0}^{N} \sum_{\substack{K \in \mathcal{K}^{n} \\ e^{0} \in \partial^{0} K}} \frac{\left|e^{0}\right|\left|e_{K}^{+}\right|}{\left|\partial^{0} K\right|}\left|\bar{\mu}_{K, e^{0}}^{n+1}-\mu_{K}^{n+1}\left(u_{K}^{n+1}\right)\right|^{2}=\mathcal{O}\left(t_{N}\right),
$$

where $t_{N}$ is defined in (5.16).

Proof. Summing the inequality (5.19) for $n=0, \ldots, N$, we observe that the first terms on each side of the inequality cancel, leaving only the terms with $n=0$ and 
$n=N$. Moreover, using the growth condition (2.5) on the divergence term gives

$$
\begin{aligned}
& \sum_{n=0}^{N} \sum_{\substack{K \in \mathcal{K}^{n} \\
e^{0} \in \partial^{0} K}} \frac{\beta_{K}^{n+1}}{2} \frac{\left|e^{0}\right|\left|e_{K}^{+}\right|}{\left|\partial^{0} K\right|}\left|\bar{\mu}_{K, e^{0}}^{n+1}-\mu_{K}^{n+1}\left(u_{K}^{n+1}\right)\right|^{2} \\
\leq & \sum_{K^{0} \in \mathcal{K}^{0}}\left|e_{K^{0}}^{-}\right|\left|V_{K}^{0}\left(\mu_{K}^{0}\left(u_{K}^{0}\right)\right)\right|+\sum_{K^{N+1} \in \mathcal{K}^{N+1}}\left|e_{K^{N}}^{+}\right|\left|V_{K}^{N+1}\left(\mu_{K}^{N+1}\left(u_{K}^{N+1}\right)\right)\right| \\
& +\sum_{n=0}^{N} \sum_{K \in \mathcal{K}^{n}}|K|\left(C_{1}+C_{2}\left|u_{K}^{j}\right|\right)+\sum_{n=0}^{N} \sum_{\substack{K \in \mathcal{K}^{n} \\
e^{0} \in \partial^{0} K}} \frac{\left|e^{0}\right|\left|e_{K}^{+}\right|}{\left|\partial^{0} K\right|} R_{K, e^{0}}^{n+1} .
\end{aligned}
$$

The last term is estimated using (5.2), (5.7), and the growth condition (2.5), yielding

$$
\begin{aligned}
\sum_{n=0}^{N} \sum_{\substack{K \in \mathcal{K}^{n} \\
e^{0} \in \partial^{0} K}} \frac{\left|e^{0}\right|\left|e_{K}^{+}\right|}{\left|\partial^{0} K\right|} R_{K, e^{0}}^{n+1} & \leq \sum_{n=0}^{N} \sum_{K \in \mathcal{K}^{n}} \operatorname{Lip} V_{K}^{n+1} \int_{K}\left|\operatorname{div}_{g} f\left(u_{K}^{n}, p\right)\right| d V_{K} \\
& \leq \sum_{n=0}^{N} \sum_{K \in \mathcal{K}^{n}}|K|\left(C_{1}+C_{2}\left|u_{K}^{j}\right|\right) .
\end{aligned}
$$

Here, we have used that $\operatorname{Lip} V_{K}^{n+1}$ is uniformly bounded, which is an easy consequence of the corresponding bounds for the flux $f$. The result now follows from (5.24) and the $L^{\infty}$ estimate in Lemma 5.2, which allows us to uniformly bound all of the terms on the right-hand side of (5.26). Note however that this bound depends, of course, on the entropy $U$. This completes the proof of Corollary 5.4.

5.3. Global entropy inequality in space and time. In this paragraph, we deduce a global entropy inequality from the local entropy inequality (5.6). This is nothing but a discrete version of the entropy inequality used to define a weak entropy solution. Given a test-function $\phi$ defined on $M$ we introduce its averages

$$
\begin{aligned}
& \phi_{e^{0}}^{n}:=\oint_{e^{0}} \phi(p) d V_{e^{0}}, \\
& \phi_{\partial^{0} K}^{n}:=\sum_{e^{0} \in \partial^{0} K} \frac{\left|e^{0}\right|}{\left|\partial^{0} K\right|} \phi_{e^{0}}^{n}=\oint_{\partial^{0} K} \phi(p) d V_{e^{0}} .
\end{aligned}
$$

We are now ready to prove the global discrete entropy inequality, which is a discrete version of the entropy inequality in Definition 2.1.

Proposition 5.5. Let $(U, F)$ be a convex entropy pair, and let $\phi$ be a non-negative test-function. Then, the function $u^{h}$ given by (3.7) satisfies the global entropy inequality

$$
\begin{aligned}
& -\sum_{n=0}^{\infty} \sum_{K \in \mathcal{K}^{n}} \int_{K} \operatorname{div}_{g}\left(F\left(u_{K}^{n}, p\right) \phi(p)\right) d V_{K}-\sum_{K \in \mathcal{K}^{0}} \int_{e_{K}^{-}} \phi_{\partial^{0} K}^{0} g_{p}\left(F\left(u_{K}^{0}, p\right), \mathbf{n}_{K, e_{K}^{-}}\right) d V_{e_{K}^{-}} \\
& +\sum_{n=0}^{\infty} \sum_{\substack{K \in \mathcal{K}^{n} \\
e^{0} \in \partial^{0} K}} \frac{\left|e^{0}\right|}{\left|\partial^{0} K\right|}\left|e_{K}^{+}\right| \phi_{e^{0}}^{n}\left(V_{K}^{n+1}\left(\tilde{\mu}_{K, e^{0}}^{n+1}\right)-V_{K}^{n+1}\left(\bar{\mu}_{K, e^{0}}^{n+1}\right)\right)
\end{aligned}
$$




$$
\begin{aligned}
\leq & \sum_{n=0}^{\infty} \sum_{\substack{K \in \mathcal{K}^{n} \\
e^{0} \in \partial^{0} K}} \frac{\left|e^{0}\right|}{\left|\partial^{0} K\right|}\left|e_{K}^{+}\right|\left(\phi_{\partial^{0} K}^{n}-\phi_{e^{0}}^{n}\right) V^{n+1}\left(\bar{\mu}_{K, e^{0}}^{n+1}\right) \\
& +\sum_{n=0}^{\infty} \sum_{\substack{K \in \mathcal{K}^{n} \\
e^{0} \in \partial^{0} K}} \int_{e^{0}}\left(\phi_{e^{0}}^{n}-\phi(p)\right) F_{e^{0}}\left(u_{K}^{n}, p\right) d V_{e^{0}} \\
& -\sum_{n=0}^{\infty} \sum_{K \in \mathcal{K}^{n}} \int_{e_{K}^{+}}\left(\phi_{\partial^{0} K}^{n}-\phi(p)\right) g\left(F\left(u_{K}^{n+1}, p\right)-F\left(u_{K}^{n}, p\right), \tilde{\mathbf{n}}_{K, e_{K}^{+}}(p)\right) d V_{e_{K}^{+}} .
\end{aligned}
$$

Proof. From the local entropy inequalities (5.6), we obtain

$$
\begin{aligned}
& \sum_{\substack{K \in \mathcal{K}^{n} \\
e^{0} \in \partial^{0} K}} \frac{\left|e^{0}\right|\left|e_{K}^{+}\right|}{\left|\partial^{0} K\right|} \phi_{e^{0}}^{n}\left(V_{K}^{n+1}\left(\bar{\mu}_{K, e^{0}}^{n+1}\right)-V_{K}^{n+1}\left(\mu_{K}^{n+1}\left(u_{K}^{n}\right)\right)\right) \\
& +\sum_{\substack{K \in \mathcal{K}^{n} \\
e^{0} \in \partial^{0} K}}\left|e^{0}\right| \phi_{e^{0}}^{n}\left(\mathbf{Q}_{K, e^{0}}\left(u_{K}^{n}, u_{K_{e^{0}}}^{n}\right)-\mathbf{Q}_{K, e^{0}}\left(u_{K}^{n}, u_{K}^{n}\right)\right) \\
\leq & \sum_{\substack{K \in \mathcal{K}^{n} \\
e^{0} \in \partial^{0} K}} \frac{\left|e^{0}\right|\left|e_{K}^{+}\right|}{\left|\partial^{0} K\right|} \phi_{e^{0}}^{n} R_{K, e^{0}}^{n+1} .
\end{aligned}
$$

Now, from the conservation property (3.10) we have that

$$
\sum_{\substack{K \in \mathcal{K}^{n} \\ e^{0} \in \partial^{0} K}}\left|e^{0}\right| \phi_{e^{0}}^{n} \mathbf{Q}_{K, e^{0}}\left(u_{K}^{n}, u_{K_{e^{0}}}^{n}\right)=0 .
$$

Also, from the consistency property (3.9), we find that

$$
\begin{aligned}
& \sum_{\substack{K \in \mathcal{K}^{n} \\
e^{0} \in \partial^{0} K}} \phi_{e^{0}}^{n}\left|e^{0}\right| \mathbf{Q}_{K, e^{0}}\left(u_{K}^{n}, u_{K}^{n}\right)=\sum_{\substack{K \in \mathcal{K}^{n} \\
e^{0} \in \partial^{0} K}} \phi_{e^{0}}^{n} \int_{e^{0}} F_{e^{0}}\left(u_{K}^{n}, p\right) d V_{e^{0}} \\
= & \sum_{\substack{K \in \mathcal{K}^{n} \\
e^{0} \in \partial^{0} K}} \int_{e^{0}} \phi(p) F_{e^{0}}\left(u_{K}^{n}, p\right) d V_{e^{0}}+\sum_{\substack{K \in \mathcal{K}^{n} \\
e^{0} \in \partial^{0} K}} \int_{e^{0}}\left(\phi_{e^{0}}^{n}-\phi(p)\right) F_{e^{0}}\left(u_{K}^{n}, p\right) d V_{e^{0}} .
\end{aligned}
$$

Next, we have that

$$
\begin{aligned}
& \sum_{\substack{K \in \mathcal{K}^{n} \\
e^{0} \in \partial^{0} K}} \frac{\left|e^{0}\right|\left|e_{K}^{+}\right|}{\left|\partial^{0} K\right|} \phi_{e^{0}}^{n} V_{K}^{n+1}\left(\bar{\mu}_{K, e^{0}}^{n+1}\right) \\
= & \sum_{\substack{K \in \mathcal{K}^{n} \\
e^{0} \in \partial^{0} K}} \frac{\left|e^{0}\right|\left|e_{K}^{+}\right|}{\left|\partial^{0} K\right|} \phi_{\partial^{0} K}^{n} V_{K}^{n+1}\left(\bar{\mu}_{K, e^{0}}^{n+1}\right)+\sum_{\substack{K \in \mathcal{K}^{n} \\
e^{0} \in \partial^{0} K}} \frac{\left|e^{0}\right|\left|e_{K}^{+}\right|}{\left|\partial^{0} K\right|}\left(\phi_{e^{0}}^{n}-\phi_{\partial^{0} K}^{n}\right) V_{K}^{n+1}\left(\bar{\mu}_{K, e^{0}}^{n+1}\right) \\
\geq & \sum_{K \in \mathcal{K}^{n}}\left|e_{K}^{+}\right| \phi_{\partial^{0} K}^{n} V_{K}^{n+1}\left(\mu_{K}^{n+1}\left(u_{K}^{n+1}\right)\right)+\sum_{\substack{K \in \mathcal{K}^{n} \\
e^{0} \in \partial^{0} K}} \frac{\left|e^{0}\right|\left|e_{K}^{+}\right|}{\left|\partial^{0} K\right|}\left(\phi_{e^{0}}^{n}-\phi_{\partial^{0} K}^{n}\right) V_{K}^{n+1}\left(\bar{\mu}_{K, e^{0}}^{n+1}\right) .
\end{aligned}
$$

Here, we have used that $V(v) \leq \sum_{j} \alpha_{j} V\left(v_{j}\right)$, for all convex functions $V$ and convex combinations $v=\sum_{j} \alpha_{j} v_{j}$, specifically used for the convex function $V_{K}^{n+1}$ and the 
convex combination (5.3). Also,

$$
\sum_{\substack{K \in \mathcal{K}^{n} \\ e^{0} \in \partial^{0} K}} \frac{\left|e^{0}\right|\left|e_{K}^{+}\right|}{\left|\partial^{0} K\right|} \phi_{e^{0}}^{n} V_{K}^{n+1}\left(\mu_{K}^{n+1}\left(u_{K}^{n}\right)\right)=\sum_{K \in \mathcal{K}^{n}}\left|e_{K}^{+}\right| \phi_{\partial^{0} K}^{n} V_{K}^{n+1}\left(\mu_{K}^{n+1}\left(u_{K}^{n}\right)\right) .
$$

Therefore, the inequality (5.28) becomes

$$
\begin{aligned}
& \sum_{K \in \mathcal{K}^{n}} \phi_{\partial^{0} K}^{n}\left|e_{K}^{+}\right|\left(V_{K}^{n+1}\left(\mu_{K}^{n+1}\left(u_{K}^{n+1}\right)\right)-V_{K}^{n+1}\left(\mu_{K}^{n+1}\left(u_{K}^{n}\right)\right)\right) \\
& -\sum_{\substack{K \in \mathcal{K}^{n} \\
e^{0} \in \partial^{0} K}} \int_{e^{0}} \phi(p) F_{e^{0}}\left(u_{K}^{n}, p\right) d V_{e^{0}} \\
\leq & \sum_{\substack{K \in \mathcal{K}^{n} \\
e^{0} \in \partial^{0} K}} \frac{\left|e^{0}\right|\left|e_{K}^{+}\right|}{\left|\partial^{0} K\right|} \phi_{e^{0}}^{n} R_{K, e^{0}}^{n+1}-\sum_{\substack{K \in \mathcal{K}^{n} \\
e^{0} \in \partial^{0} K}} \frac{\left|e^{0}\right|\left|e_{K}^{+}\right|}{\left|\partial^{0} K\right|}\left(\phi_{e^{0}}^{n}-\phi_{\partial^{0} K}^{n}\right) V_{K}^{n+1}\left(\bar{\mu}_{K, e^{0}}^{n+1}\right) \\
& +\sum_{\substack{K \in \mathcal{K}^{n} \\
e^{0} \in \partial^{0} K}} \int_{e^{0}}\left(\phi_{e^{0}}^{n}-\phi(p)\right) F_{e^{0}}\left(u_{K}^{n}, p\right) d V_{e^{0}}=: A^{h}+B^{h}+C^{h} .
\end{aligned}
$$

The first term in (5.29) can be written as

$$
\begin{aligned}
& \sum_{K \in \mathcal{K}^{n}} \phi_{\partial^{0} K}^{n}\left|e_{K}^{+}\right|\left(V_{K}^{n+1}\left(\mu_{K}^{n+1}\left(u_{K}^{n+1}\right)\right)-V_{K}^{n+1}\left(\mu_{K}^{n+1}\left(u_{K}^{n}\right)\right)\right) \\
= & \sum_{K \in \mathcal{K}^{n}} \int_{e_{K}^{+}} \phi(p) g\left(F\left(u_{K}^{n+1}, p\right)-F\left(u_{K}^{n}, p\right), \tilde{\mathbf{n}}_{K, e_{K}^{+}}(p)\right) d V_{e_{K}^{+}} \\
& +\sum_{K \in \mathcal{K}^{n}} \int_{e_{K}^{+}}\left(\phi_{\partial^{0} K}^{n}-\phi(p)\right) g\left(F\left(u_{K}^{n+1}, p\right)-F\left(u_{K}^{n}, p\right), \tilde{\mathbf{n}}_{K, e_{K}^{+}}(p)\right) d V_{e_{K}^{+}} .
\end{aligned}
$$

Combining this result with the identity

$$
\begin{aligned}
& \int_{K} \operatorname{div}_{g}(F(u, p) \phi(p)) d V_{K}=\int_{\partial K} \phi(p) g\left(F(u, p), \tilde{\mathbf{n}}_{\partial K}\right) d V_{\partial K} \\
= & \int_{e_{K}^{+}} \phi(p) g\left(F(u, p), \tilde{\mathbf{n}}_{K, e_{K}^{+}}\right) d V_{e_{K}^{+}}+\int_{e_{K}^{-}} \phi(p) g\left(F(u, p), \tilde{\mathbf{n}}_{K, e_{K}^{-}}\right) d V_{e_{K}^{-}} \\
& +\sum_{e^{0} \in \partial^{0} K} \int_{e^{0}} \phi(p) F_{e^{0}}(u, p) d V_{e^{0}}
\end{aligned}
$$

(with $u=u_{K}^{n}$ ) and in view of (5.29) we see that

$$
\begin{aligned}
& -\sum_{K \in \mathcal{K}^{n}} \int_{K} \operatorname{div}_{g}\left(F\left(u_{K}^{n}, p\right) \phi(p)\right) d V_{K} \\
\leq & A^{h}+B^{h}+C^{h} \\
& -\sum_{K \in \mathcal{K}^{n}}\left(\int_{e_{K}^{+}} \phi(p) g\left(F\left(u_{K}^{n+1}, p\right), \tilde{\mathbf{n}}_{K, e_{K}^{+}}(p)\right) d V_{e_{K}^{+}}+\int_{e_{K}^{-}} \phi(p) g\left(F\left(u_{K}^{n}, p\right), \tilde{\mathbf{n}}_{K, e_{K}^{-}}\right) d V_{e_{K}^{-}}\right) \\
& -\sum_{K \in \mathcal{K}^{n}} \int_{e_{K}^{+}}\left(\phi_{\partial^{0} K}^{n}-\phi(p)\right) g\left(F\left(u_{K}^{n+1}, p\right)-F\left(u_{K}^{n}, p\right), \tilde{\mathbf{n}}_{K, e_{K}^{+}}(p)\right) d V_{e_{K}^{+}}
\end{aligned}
$$


The inequality (5.27) is now obtained by summation in $n$. First, the (summed) terms $A, B, C$ give the three terms on the right-hand side of (5.27) while, in the first sum above, terms cancel out two at a time and on the second term remains in the left-hand side of (5.27). This completes the proof of Proposition 5.5.

\section{Proof of convergence}

This section contains a proof of the convergence of the finite volume method, and is based on the framework of measure-valued solutions to conservation laws, introduced by DiPerna [11] and extended to manifolds by Ben-Artzi and LeFloch [2]. The basic strategy will be to rely on the discrete entropy inequality (5.27) as well as on the entropy dissipation estimate (5.25), in order to check that any Young measure associated with the approximate solution is a measure-valued solution to the Cauchy problem under consideration. In turn, by the uniqueness result for measure-valued solutions it follows that, in fact, this solution is the unique weak entropy solution of the problem under consideration.

In the following, for the sake of simplicity, we denote by $\mathbf{M}$ our domain of discretization, which is not necessarily the whole manifold. Since the sequence $u^{h}$ is uniformly bounded in $L^{\infty}(\mathbf{M})$, we can associate a subsequence and a Young measure $\nu: \mathbf{M} \rightarrow \operatorname{Prob}(\mathbb{R})$, which is a family of probability measures in $\mathbb{R}$ parametrized by $p \in \mathbf{M}$. The Young measure allows us to determine all weak-* limits of composite functions $a\left(u^{h}\right)$, for arbitrary real continuous functions $a$, according to the following property :

$$
a\left(u^{h}\right) \stackrel{*}{\rightarrow}\langle\nu, a\rangle \quad \text { as } h \rightarrow 0
$$

where we use the notation $\langle\nu, a\rangle:=\int_{\mathbb{R}} a(\lambda) d \nu(\lambda)$.

In view of the above property, the passage to the limit in the left-hand side of (5.27) is (almost) immediate. The uniqueness theorem $[11,2]$ tells us that once we know that $\nu$ is a measure-valued solution to the conservation law, we can prove that the support of each probability measure $\nu_{p}$ actually reduces to a single value $u(p)$, if the same is true on $\mathcal{H}_{0}$, that is, $\nu_{p}$ is the Dirac measure $\delta_{u(p)}$. It is then standard to deduce that the convergence in (6.1) is actually strong, and that, in particular, $u^{h}$ converges strongly to $u$ which in turn is the unique entropy solution of the Cauchy problem under consideration.

Lemma 6.1. Let $\nu_{p}$ be the Young measure associated with the sequence $u^{h}$. Then, for every convex entropy pair $(U, F)$ and every non-negative test-function $\phi$ defined on $M$ with compact support, we have

$$
\begin{aligned}
& -\int_{M}\left\langle\nu_{p}, \operatorname{div}_{g} F(\cdot, p)\right\rangle \phi(p)+g\left(\left\langle\nu_{p}, F(\cdot, p)\right\rangle, \nabla \phi\right) d V_{M} \\
& -\int_{\mathcal{H}_{0}} \phi(p) g\left(\left\langle\nu_{p}, F(\cdot, p)\right\rangle, \mathbf{n}_{\mathcal{H}_{0}}\right) d V_{\mathcal{H}_{0}}+\int_{M} \phi(p)\left\langle\nu_{p}, U^{\prime}(\cdot) \operatorname{div}_{g} f(\cdot, p)\right\rangle d V_{M} \leq 0 .
\end{aligned}
$$

The following lemma is easily deduced from the corresponding result in the Euclidean space, by relying on a system of local coordinates. This result will be useful when analyzing the approximation.

Lemma 6.2. Let $G: M \rightarrow \mathbb{R}$ be a smooth function, and let e be a submanifold of $M$. Then, there exists a point $p_{e}$ (not necessarily in e), the center of mass of e, such that

$$
\left|\oint_{e} G(p) d V_{e}-G\left(p_{e}\right)\right| \leq \operatorname{diam}(e)^{2}\|G\|_{C^{2}(e)} .
$$


We are now in position to complete the proof of the main theorem of this paper.

Proof. [Proof of Theorem 3.3.] Due to (6.2), we have for all convex entropy pairs $(U, F)$,

$$
\operatorname{div}_{g}\langle\nu, F(\cdot)\rangle-\left\langle\nu,\left(\operatorname{div}_{g} F\right)(\cdot)\right\rangle+\left\langle\nu, U^{\prime}(\cdot)\left(\operatorname{div}_{g} f\right)(\cdot)\right\rangle \leq 0
$$

in the sense of distributions in $M$. Since on the initial hypersurface $\mathcal{H}_{0}$ the (trace of the) Young measure $\nu$ coincides with the Dirac mass $\delta_{u_{0}}$ (because $u_{0}$ is a bounded function), from the theory in [2] there exists a unique function $u \in L^{\infty}(M)$ such that the measure $\nu$ remains the Dirac mass $\delta_{u}$ for all Cauchy hypersurfaces $\mathcal{H}_{t}, 0 \leq t \leq T$. Moreover, this implies that the approximations $u^{h}$ converge strongly to $u$ at least on compact sets. This concludes the proof.

Proof. [Proof of Lemma 6.1.] The proof consists of passing inequality (5.27) to the limit and using property (6.1) of the Young measure. First, note that the first term on the left-hand side of inequality (5.27) converges immediately to the first integral term of (6.2). Next, take the second term of (5.27). Using the fact that $\phi_{\partial^{0} K}^{n}-\phi(p)=\mathcal{O}(\tau+h)$, we see that this term converges to the second integral term in $(6.2)$.

Next, we will prove that the third term on the left-hand side of (5.27) converges to the last term in (6.2). Observe first that

$$
\tilde{\mu}_{K, e^{0}}^{n+1}-\bar{\mu}_{K, e^{0}}^{n+1}=\frac{1}{\left|e_{K}^{+}\right|} \int_{K} \operatorname{div}_{g} f\left(u_{K}^{n}, p\right) d V_{K}
$$

Therefore, we obtain

$$
\begin{aligned}
& -\sum_{n=0}^{\infty} \sum_{\substack{K \in \mathcal{K}^{n} \\
e^{0} \in \partial^{0} K}} \frac{\left|e^{0}\right|}{\left|\partial^{0} K\right|}\left|e_{K}^{+}\right| \phi_{e^{0}}^{n}\left(V_{K}^{n+1}\left(\tilde{\mu}_{K, e^{0}}^{n+1}\right)-V_{K}^{n+1}\left(\bar{\mu}_{K, e^{0}}^{n+1}\right)\right) \\
= & \sum_{n=0}^{\infty} \sum_{\substack{K \in \mathcal{K}^{n} \\
e^{0} \in \partial^{0} K}} \frac{\left|e^{0}\right|}{\left|\partial^{0} K\right|} \phi_{e^{0}}^{n}\left(\partial_{\mu} V_{K}^{n+1}\left(\tilde{\mu}_{K, e^{0}}^{n+1}\right) \int_{K} \operatorname{div}_{g} f\left(u_{K}^{n}, p\right) d V_{K}+\left|e_{K}^{+}\right| \mathcal{O}\left(\tau^{2}\right)\right) \\
= & \sum_{n=0}^{\infty} \sum_{\substack{K \in \mathcal{K}^{n} \\
e^{0} \in \partial^{0} K}} \frac{\left|e^{0}\right|}{\left|\partial^{0} K\right|} \phi_{e^{0}}^{n}\left(\left(\partial_{\mu} V_{K}^{n+1}\left(\tilde{\mu}_{K, e^{0}}^{n+1}\right)-U^{\prime}\left(u_{K}^{n+1}\right)\right) \int_{K} \operatorname{div}_{g} f\left(u_{K}^{n}, p\right) d V_{K}\right. \\
& \left.\quad+\left|e_{K}^{+}\right| \mathcal{O}\left(\tau^{2}\right)+U^{\prime}\left(u_{K}^{n+1}\right) \int_{K} \operatorname{div}_{g} f\left(u_{K}^{n}, p\right) d V_{K}\right) .
\end{aligned}
$$

Now, note that from the expression of $V$ (see (5.8)),

$$
\begin{aligned}
\partial_{\mu} V_{K}^{n+1}\left(\tilde{\mu}_{K, e^{0}}^{n+1}\right)-U^{\prime}\left(u_{K}^{n+1}\right) & =U^{\prime}\left(\left(\mu_{K}^{n+1}\right)^{-1}\left(\tilde{\mu}_{K, e^{0}}^{n+1}\right)\right)-U^{\prime}\left(u_{K}^{n+1}\right) \\
& \leq \sup U^{\prime \prime} \max _{n, K} \operatorname{Lip}\left(\mu_{K}^{n}\right)^{-1}\left|\tilde{\mu}_{K, e^{0}}^{n+1}-\mu_{K}^{n+1}\right|,
\end{aligned}
$$


and so, using the $L^{\infty}$ bound (5.15) and the growth condition (2.5), we find

$$
\begin{aligned}
& \sum_{n=0}^{\infty} \sum_{\substack{K \in \mathcal{K}^{n} \\
e^{0} \in \partial^{0} K}} \frac{\left|e^{0}\right|}{\left|\partial^{0} K\right|} \phi_{e^{0}}^{n}\left(\partial_{\mu} V_{K}^{n+1}\left(\tilde{\mu}_{K, e^{0}}^{n+1}\right)-U^{\prime}\left(u_{K}^{n+1}\right)\right) \int_{K} \operatorname{div}_{g} f\left(u_{K}^{n}, p\right) d V_{K} \\
\lesssim & \sum_{n=0}^{\infty} \sum_{\substack{K \in \mathcal{K}^{n} \\
e^{0} \in \partial^{0} K}} \frac{\left|e^{0}\right|}{\left|\partial^{0} K\right|} \phi_{e^{0}}^{n}|K|\left|\tilde{\mu}_{K, e^{0}}^{n+1}-\mu_{K}^{n+1}\right| .
\end{aligned}
$$

Applying the Cauchy-Schwarz inequality and the entropy dissipation estimate (5.25), we find that this term tends to zero with $h$. Note that property (5.24) is easily seen to be verified due to the smoothness of the functions $V_{K}^{n}$. We are left with the term

$$
\sum_{n=0}^{\infty} \sum_{K \in \mathcal{K}^{n}} \phi_{\partial^{0} K}^{n} U^{\prime}\left(u_{K}^{n+1}\right) \int_{K} \operatorname{div}_{g} f\left(u_{K}^{n}, p\right) d V_{K}
$$

which is easily seen to be of the form

$$
\sum_{n=0}^{\infty} \sum_{K \in \mathcal{K}^{n}} U^{\prime}\left(u_{K}^{n}\right) \int_{K} \phi(p) \operatorname{div}_{g} f\left(u_{K}^{n}, p\right) d V_{K}+\mathcal{O}(h) \rightarrow \int_{M}\left\langle\nu_{p}, U^{\prime}(\cdot) \operatorname{div}_{g} f(\cdot, p)\right\rangle d V_{M} .
$$

It remains to check that the terms on the right-hand side of (5.27) tend to zero with $h$. Namely, the first term on the right-hand side can be written as

$$
\begin{aligned}
& \sum_{n=0}^{\infty} \sum_{\substack{K \in \mathcal{K}^{n} \\
e^{0} \in \partial^{0} K}} \frac{\left|e^{0}\right|}{\left|\partial^{0} K\right|}\left|e_{K}^{+}\right|\left(\phi_{\partial^{0} K}^{n}-\phi_{e^{0}}^{n}\right) V^{n+1}\left(\bar{\mu}_{K, e^{0}}^{n+1}\right) \\
= & \sum_{n=0}^{\infty} \sum_{\substack{K \in \mathcal{K}^{n} \\
e^{0} \in \partial^{0} K}} \frac{\left|e^{0}\right|}{\left|\partial^{0} K\right|}\left|e_{K}^{+}\right|\left(\phi_{\partial^{0} K}^{n}-\phi_{e^{0}}^{n}\right)\left(V^{n+1}\left(\bar{\mu}_{K, e^{0}}^{n+1}\right)-V^{n+1}\left(\mu_{K}^{n+1}\left(u_{K}^{n+1}\right)\right)\right) \\
= & o(1),
\end{aligned}
$$

by the Cauchy-Schwarz inequality and the entropy dissipation estimate (5.25). Next, the second term on the right-hand side of (5.27) satisfies

$$
\begin{aligned}
& \sum_{n=0}^{\infty} \sum_{\substack{K \in \mathcal{K}^{n} \\
e^{0} \in \partial^{0} K}} \int_{e^{0}}\left(\phi_{e^{0}}^{n}-\phi(p)\right) F_{e^{0}}\left(u_{K}^{n}, p\right) d V_{e^{0}} \\
= & \sum_{n=0}^{\infty} \sum_{\substack{K \in \mathcal{K}^{n} \\
e^{0} \in \partial^{0} K}} \int_{e^{0}}\left(\phi_{e^{0}}^{n}-\phi(p)\right)\left(F_{e^{0}}\left(u_{K}^{n}, p\right)-\oint_{e^{0}} F_{e^{0}}\left(u_{K}^{n}, q\right) d q\right) d V_{e^{0}}
\end{aligned}
$$

which, in view of the regularity of $\phi$ and $F$, is bounded by $\sum_{n=0}^{\infty} \sum_{K \in \mathcal{K}^{n}}\left|\partial^{0} K\right| \mathcal{O}\left(\tau_{K}+\right.$ $h)^{2}$. Using the CFL condition (3.14) and property (3.13), we can further bound this term by

$$
\sum_{n=0}^{\infty} \sum_{K \in \mathcal{K}^{n}}\left|e_{K}^{+}\right| \mathcal{O}\left(\tau_{K}\right)\left(\mathcal{O}\left(\tau_{K}+h\right)+\mathcal{O}\left(h^{2} / \tau_{K}\right)\right)=o(1)
$$


and so only the term

$$
A^{h}(\phi):=-\sum_{n=0}^{N} \sum_{K \in \mathcal{K}^{n}} \int_{e_{K}^{+}}\left(\phi_{\partial^{0} K}^{n}-\phi(p)\right) g\left(F\left(u_{K}^{n+1}, p\right)-F\left(u_{K}^{n}, p\right), \tilde{\mathbf{n}}_{K, e_{K}^{+}}(p)\right) d V_{e_{K}^{+}}
$$

remains to be controlled. Here, we will use that our triangulation is admissible, in the sense of Definition 3.2. First of all, by integrating by parts we rewrite it as

$$
\begin{array}{r}
A^{h}(\phi)=\sum_{n=1}^{\infty} \sum_{K \in \mathcal{K}^{n}} \int_{e_{K}^{-}}\left(\phi_{\partial^{0} K}^{n-1}-\phi(p)\right) g\left(F\left(u_{K}^{n}, p\right), \mathbf{n}_{K, e_{K}^{-}}(p)\right) d V_{e_{K}^{+}} \\
+\int_{e_{K}^{+}}\left(\phi_{\partial^{0} K}^{n}-\phi(p)\right) g\left(F\left(u_{K}^{n}, p\right), \mathbf{n}_{K, e_{K}^{+}}(p)\right) d V_{e_{K}^{+}}
\end{array}
$$

plus a boundary term for $n=0$ which easily tends to zero with $h$. Next, using Lemma 6.2 and equation (3.1), one may replace $\phi_{\partial^{0} K}^{n}$ by $\phi\left(p_{K}^{0}\right)$ and $\phi_{\partial^{0} K}^{-}$by $\phi\left(p_{K^{n-1}}^{0}\right)$, where $p_{K^{j}}^{0}$ denotes the center of $\partial^{0} K^{j}$, with an error term of the form $C h\|\phi\|_{C^{2}}\|F\|_{L^{\infty}}$. Next, we replace (and similarly for $\left.e_{K}^{-}\right) \phi(p) g\left(F\left(u_{K}^{n}, p\right), \mathbf{n}_{K, e_{K}^{+}}(p)\right.$ ) with $\phi\left(p_{K}^{+}\right) g\left(F\left(u_{K}^{n}, p_{K}^{+}\right), \mathbf{n}_{K, e_{K}^{+}}\left(p_{K}^{+}\right)\right)$. Using property (3.1), the corresponding error term is seen to be of the form $C h\|\phi\|_{C^{2}}\|F\|_{C^{2}}$. The generic constants $C$ do not depend on $h$ nor $\phi$.

We have

$$
\begin{aligned}
\left|A^{h}(\phi)\right| \leq & \left|\sum_{n=1}^{\infty} \sum_{K \in \mathcal{K}^{n}}\right| e_{K}^{+} \mid\left(\phi\left(p_{K}^{0}\right)-\phi\left(p_{K}^{+}\right)\right) g\left(F\left(u_{K}^{n}, p_{K}^{+}\right), \mathbf{n}_{K, e_{K}^{+}}\left(p_{K}^{+}\right)\right) \\
& \quad+\left|e_{K}^{-}\right|\left(\phi\left(p_{K^{-}}^{0}\right)-\phi\left(p_{K^{-}}^{+}\right)\right) g\left(F\left(u_{K}^{n}, p_{K^{-}}^{+}\right), \mathbf{n}_{K, e_{K}^{-}}\left(p_{K^{-}}^{+}\right)\right) \mid \\
& +h\|\phi\|_{C^{2}}\left(\|F\|_{L^{\infty}}+\|F\|_{C^{2}}\right) .
\end{aligned}
$$

Now, performing a Taylor expansion of $\phi$ and using the definition of $\mathbf{w}_{K}$ (recall that $\mathbf{w}_{K}$ is the future-oriented vector at $p_{K}^{+}$tangent to the geodesic connecting $p_{K}^{+}$and $p_{K}^{0}$ ) we find, for instance,

$$
\phi\left(p_{K}^{0}\right)-\phi\left(p_{K}^{+}\right)=g\left(\mathbf{w}_{K}, \nabla \phi\left(p_{K}^{+}\right)\right)+\mathcal{O}\left(h^{2}\right) .
$$

Therefore, by the definition of $\mathcal{E}(K)$ and using (3.15), we may express this conclusion by using the local deviation of the triangulation,

$$
\begin{aligned}
\left|A^{h}(\phi)\right| \leq & \left|\sum_{n=1}^{\infty} \sum_{K \in \mathcal{K}^{n}}\right| e_{K}^{+} \mid g\left(\mathbf{w}_{K}, \nabla \phi\left(p_{K}^{+}\right)\right) g\left(F\left(u_{K}^{n}, p_{K}^{+}\right), \mathbf{n}_{K, e_{K}^{+}}\left(p_{K}^{+}\right)\right) \\
& \quad+\left|e_{K}^{-}\right| g\left(\mathbf{w}_{K^{-}}, \nabla \phi\left(p_{K^{-}}^{+}\right)\right) g\left(F\left(u_{K}^{n}, p_{K^{-}}^{+}\right), \mathbf{n}_{K, e_{K}^{-}}\left(p_{K^{-}}^{+}\right)\right) \mid \\
& +C h\|\phi\|_{C^{2}}\left(\|F\|_{L^{\infty}}+\|F\|_{C^{2}}\right) \\
\leq & \sum_{K \in \mathcal{T}^{h}}\left(|K| \mathcal{E}(K)-\left|K^{-}\right| \mathcal{E}\left(K^{-}\right)\right)\left(\nabla \phi, F\left(u^{h}\right)\right)+C h\|\phi\|_{C^{2}}\left(\|F\|_{L^{\infty}}+\|F\|_{C^{2}}\right) \\
\leq & \eta(h)\|\phi\|_{C^{1}}\|F\|_{L^{\infty}}+C h\|\phi\|_{C^{2}}\left(\|F\|_{L^{\infty}}+\|F\|_{C^{2}}\right)
\end{aligned}
$$

which tends to zero since $\eta(h) \rightarrow 0$. This completes the proof of Lemma 6.1 . 
Acknowledgement. The authors were partially supported by the Agence Nationale de la Recherche (A.N.R.) through the grant 06-2-134423 entitled "Mathematical Methods in General Relativity" (MATH-GR), and by the Centre National de la Recherche Scientifique (CNRS). The first author (P.A.) was also supported by the FCT-Fundação para a Ciência e Tecnologia (Portuguese Foundation for Science and Technology) through the grant SFRH/BD/17271/2004.

\section{REFERENCES}

[1] P. Amorim, M. Ben-Artzi and P.G. LeFloch, Hyperbolic conservation laws on manifolds: total variation estimates and the finite volume method, Meth. Appl. Anal., 12, 291-324, 2005.

[2] M. Ben-Artzi and P.G. LeFloch, The well-posedness theory for geometry compatible, hyperbolic conservation laws on manifolds, Ann. Inst. H. Poincaré : Nonlin. Anal., 24, 989-1008, 2007.

[3] M. Ben-Artzi, J. Falcovitz and P.G. LeFloch, Hyperbolic conservation laws on the sphere. A geometry-compatible finite volume scheme, preprint, 2008.

[4] B. Cockburn, F. Coquel and P.G. LeFloch, An error estimate for high-order accurate finite volume methods for scalar conservation laws, AHCRC Inst., Minneapolis, 91-20, 1991.

[5] B. Cockburn, F. Coquel and P.G. LeFloch, Convergence of finite volume methods, I.M.A. Series \# 771, Minneapolis, February, preprint, 1991.

[6] B. Cockburn, F. Coquel and P.G. LeFloch, An error estimate for finite volume methods for multidimensional conservation laws, Math. Comp., 63, 77-103, 1994.

[7] B. Cockburn, F. Coquel and P.G. LeFloch, Convergence of finite volume methods for multidimensional conservation laws, SIAM J. Numer. Anal., 32, 687-705, 1995.

[8] F. Coquel and P.G. LeFloch, Convergence of finite difference schemes for conservation laws in several space dimensions, C.R. Acad. Sci. Paris Ser. I, 310, 455-460, 1990.

[9] F. Coquel and P.G. LeFloch, Convergence of finite difference schemes for conservation laws in several space dimensions: a general theory, SIAM J. Numer. Anal., 30, 675-700, 1993.

[10] F. Coquel and P.G. LeFloch, Convergence of finite difference schemes for conservation laws in several space dimensions: the corrected antidiffusive flux approach, Math. Comp., 57, 169-210, 1991.

[11] R.J. DiPerna, Measure-valued solutions to conservation laws, Arch. Rational Mech. Anal., 88, 223-270, 1985.

[12] R. Eymard, T. Gallouët and R. Herbin, The finite volume method, in Handbook of Numerical Analysis, North-Holland, Amsterdam, Vol. VII, 713-1020, 2000.

[13] D. Kröner, Finite volume schemes in multidimensions, in Numerical analysis, 1997 (Dundee), Pitman Res. Notes Math. Ser., 380, Longman, Harlow, 179-192, 1998.

[14] D. Kröner, S. Noelle and M. Rokyta, Convergence of higher-order upwind finite volume schemes on unstructured grids for scalar conservation laws with several space dimensions, Numer. Math., 71, 527-560, 1995.

[15] S.N. Kruzkov, First-order quasilinear equations with several space variables, Math. USSR Sb., 10, 217-243, 1970.

[16] P.G. LeFloch, W. Neves and B. Okutmustur, Hyperbolic conservation laws on manifolds. Error estimate for finite volume schemes, Acta Math. Sinica, 2009.

[17] P.G. LeFloch and B. Okutmustur, Conservation laws on manifolds with limited regularity, C.R. Acad. Sc. Paris, Ser. I, 346, 539-543, 2008.

[18] P.G. LeFloch and B. Okutmustur, Hyperbolic conservation laws on spacetimes. A finite volume scheme based on differential forms, Far East J. Math. Sc., 2008. (See also arXiv:0810.0255.)

[19] E.Y. Panov, On the Cauchy problem for a first-order quasilinear equation on a manifold, Differ. Equ., 33, 257-266, 1997.

[20] J.A. Rossmanith, D.S. Bale and R.J. LeVeque, A wave propagation algorithm for hyperbolic systems on curved manifolds, J. Comput. Phys., 199, 631-662, 2004

[21] A. Szepessy, Convergence of a shock-capturing streamline diffusion finite element method for a scalar conservation law in two space dimensions, Math. Comp., 53, 527-545, 1989.

[22] A. Szepessy, Convergence of a streamline diffusion finite element method for scalar conservation laws with boundary conditions, RAIRO Modél. Math. Anal. Numér., 25, 749-782, 1991.

[23] E. Tadmor, Approximate solutions of nonlinear conservation laws, in Advanced Numerical Approximation of Nonlinear Hyperbolic Equations, (Cetraro, 1997), Lecture Notes in Math., 
1697, Springer, Berlin, 1-149, 1998.

[24] E. Tadmor, M. Rascle and P. Bagnerini, Compensated compactness for 2D conservation laws, J. Hyperbolic Differ. Equ., 2, 697-712, 2005.

[25] M. Westdickenberg and S. Noelle, A new convergence proof for finite volume schemes using the kinetic formulation of conservation laws, SIAM J. Numer. Anal., 37, 742-757, 2000. 